\title{
无铅非线性介电储能陶瓷：现状与挑战
}

\author{
杜红亮 ${ }^{1}$, 杨泽田 ${ }^{1}$, 高 峰 $^{2}$, 靳 立 $^{3}$, 程花蕾 ${ }^{4}$, 屈绍波 ${ }^{1}$
}

(1. 空军工程大学 理学院, 西安 710051；2. 西北工业大学 凝固技术国家重点实验室，西安 710072; 3. 西安交通 大学电子陶瓷与器件教育部重点实验室, 西安 710049; 4. 宝鸡文理学院 化学与化工学院, 宝鸡 721013)

摘 要: 相对于聚合物等储能介质材料, 介电陶瓷具有温度稳定性好和循环寿命长的优点, 是制备脉冲功率储能电 容器的优秀候选材料。但目前介电陶瓷的储能密度相对较低, 不能满足脉冲功率设备小型化的要求。因此, 如何显 著提高介电陶瓷的储能密度成为近年来功能陶瓷研究的热点之一。本文首先介绍了介电储能电容器对陶瓷材料性 能的要求, 然后结合本课题组的研究工作, 评述了 $\mathrm{BaTiO}_{3}$ 基、 $\mathrm{BiFeO}_{3}$ 基、 $\left(\mathrm{K}_{0.5} \mathrm{Na}_{0.5}\right) \mathrm{NbO}_{3}$ 基无铅弛豫铁电陶瓷和 $\left(\mathrm{Bi}_{0.5} \mathrm{Na}_{0.5}\right) \mathrm{TiO}_{3}$ 基、 $\mathrm{AgNbO}_{3}$ 基无铅反铁电陶瓷储能特性的研究现状, 重点阐述了不同材料体系的组分设计思路及 相关储能特性，分析了无铅非线性介电储能陶瓷所面临的机遇和挑战，指出了应对策略。最后，展望了下一步的研 究方向和内容。

关 键 词: 无铅陶瓷; 储能密度; 击穿场强; 极化差值; 综述

中图分类号: TM534 文献标识码: A

\section{Lead-free Nonlinear Dielectric Ceramics for Energy Storage Applications: Current Status and Challenges}

\author{
DU Hong-Liang ${ }^{1}$, YANG Ze-Tian ${ }^{1}$, GAO Feng ${ }^{2}, \mathrm{JIN} \mathrm{Li}^{3}$, CHENG Hua-Lei ${ }^{4}$, QU Shao-Bo ${ }^{1}$
}

(1. Science College, Air Force Engineering University, Xi'an 710051, China; 2. State Key Laboratory of Solidification Processing, Northwestern Polytechnical University, Xi'an 710072, China; 3. Electronic Materials Research Laboratory, Key Laboratory of the Ministry of Education \& International Center for Dielectric Research, Xi'an Jiaotong University, Xi'an 710049, China; 4. Department of Chemistry, Baoji University of Arts and Science, Baoji 721013, China)

\begin{abstract}
Compared to polymers and their nanocomposites, dielectric ceramics are considered as promising candidates for the pulsed-power devices because of their excellent temperature stability and good anti-fatigue characteristic. Nevertheless, relatively low energy storage density is the main disadvantage for dielectric ceramics, which does not meet the requirement of miniaturization for pulsed-power devices. Therefore, how to improve the energy storage density of dielectric ceramics has become one of hot topics on the research of functional ceramics in recent years. In this paper, the basic principle of the capacitor for electric energy storage was introduced firstly and then the research advances of $\mathrm{BaTiO}_{3}$-based, $\mathrm{BiFeO}_{3}$-based, $\left(\mathrm{K}_{0.5} \mathrm{Na}_{0.5}\right) \mathrm{NbO}_{3}$-based lead-free relaxor ceramics and $\left(\mathrm{Bi}_{0.5} \mathrm{Na}_{0.5}\right) \mathrm{TiO}_{3}$-based, and $\mathrm{AgNbO}_{3}$-based lead-free anti-ferroelectric ceramics were reviewed based on our group's research, in which the composition design strategies of different material systems were especially summarized. Finally, the opportunities and challenges of lead-free nonlinear energy-storage ceramics were analyzed, and the coping strategies as well as the future development direction were also proposed.
\end{abstract}

收稿日期: 2017-12-12；收到修改稿日期：2018-02-06

基金项目: 国家自然科学基金(21501007); 陕西省自然科学基金(2017JM5016); 西北工业大学凝固技术国家重点实验室开 放课题(SKLSP201709, SKLSP201718)

National Science Foundation of China (21501007); Natural Science Basis Research Plan in Shaanxi Province of China (2017JM5016); The Fund of the State Key Laboratory of Solidification Processing in NWPU(SKLSP201709, SKLSP201718)

作者简介: 杜红亮(1976-), 男, 副教授. E-mail: duhongliang@126.com 
Key words: lead-free ceramics; energy storage density; dielectric breakdown strength; polarization difference; review

介电储能电容器具有功率密度 $\left(\sim 10^{8} \mathrm{~W} / \mathrm{kg}\right)$ 高、 充放电速度快 $(<1 \mu \mathrm{s})$ 和循环寿命长 $(\sim 5$ 万次 $)$ 的优点, 在核物理与技术、新能源发电系统、医用手术激光、 混合动力汽车、石油天然气勘探和定向能武器等领 域得到广泛应用, 成为脉冲功率设备中最关键的元 件之一 ${ }^{[1-5]}$ 。然而, 目前介电储能电容器的储能密度 $\left(W_{\mathrm{rec}}\right)$ 相对较低 $\left(10^{-2} \sim 10^{-1} \mathrm{Wh} / \mathrm{kg}\right)^{[6-8]}$, 不能满足脉冲 功率器件集成化、轻量化和小型化的需求。因此, 如 何显著提高储能电容器用介质材料的 $W_{\text {rec }}$ 成为当前 功能材料领域的研究热点之一[9-11]。2015 年国家重 点基础研究发展计划和重大科学研究计划把高储能 密度无机电介质材料作为重要支持方向, 因此, 高 储能密度介电陶瓷的研究具有重要意义, 是符合 “国家目标” 的前沿热点研究。

目前用于介电储能电容器的介质材料主要包括 聚合物、陶瓷-聚合物复合材料、玻璃、玻璃陶瓷和 陶瓷五大类 ${ }^{[12-16]}$ 。相对于其他储能介质材料, 介电 陶瓷具有中等击穿场强 $\left(E_{\mathrm{b}}\right) 、$ 较低的介电损耗, 优良 的温度稳定性和抗疲劳特性, 可以更好地满足航空 航天、石油钻井、电磁脉冲武器等领域对储能电容 器的需求 ${ }^{[17-18]}$ 。因而, 陶瓷介质材料被认为是制备 耐高温介电脉冲功率储能电容器的优选材料。

根据材料厚度的不同，陶瓷介质通常可分为薄 膜(厚度小于 $1 \mu \mathrm{m}$ )、厚膜(厚度介于 $1 \mu \mathrm{m}$ 和 $100 \mu \mathrm{m}$ 之间)和块体(厚度大于 $100 \mu \mathrm{m}$ )三类。薄膜材料因厚 度较小, 减少了材料中的缺陷, 因而具有较高的 $E_{\mathrm{b}}$ 和 $W_{\mathrm{rec}}{ }^{[19-20]}$ 。但由于厚度的限制, 薄膜的绝对储存 能量较低。同时薄膜材料还存在制备过程复杂, 难 以制备大尺寸样品, 制备环境条件要求高等缺点, 使其应用限制在某些特定领域，如医疗上用的电击 器 ${ }^{[12]}$ 。厚膜材料厚度介于薄膜和块体之间, 兼具二 者的优点。相对于块体材料, 厚膜材料的 $E_{\mathrm{b}}$ 较高, 尺寸较小, 便于设备的集成化和小型化。相对于薄 膜而言，厚膜材料可以制备成多层结构，具有更大 的厚度, 储存的总能量也相对较高 ${ }^{[21-22]}$ 。块体材料 具有制备过程简单、制备成本低、机械强度高、热 稳定性好和储存总能量高的优点。另外, 如果陶瓷 介质的块体形式具有良好的储能特性，那么以相同 材料体系制备的薄膜或厚膜将具有更大 $W_{\mathrm{rec}}{ }^{[23-24]}$ 。 也就是说, 块体材料储能特性的研究可以为薄膜和 厚膜材料的研究提供前期探索。此外, 铅不仅危害 人体而且污染环境, 因此无铅电子材料已成为当今 的发展趋势 ${ }^{[25-26]}$ 。更重要的是, 无铅介电陶瓷的密度 $\left(<5.5 \mathrm{~g} / \mathrm{cm}^{3}\right)$ 通常小于铅基陶瓷的密度 $\left(\sim 7.5 \mathrm{~g} / \mathrm{cm}^{3}\right)$ ，在 相同 $W_{\mathrm{rec}}$ 的情况下, 更容易满足脉冲功率设备对储 能电容器“轻量化”的要求。因此, 寻求具有高 $W_{\mathrm{rec}}$ 的无铅块体材料具有重要意义。

近年来，关于储能介质材料的综述主要集中在 聚合物和陶瓷-聚合物复合材料 ${ }^{[4-5,27-28]}$, 针对无铅 非线性介电储能陶瓷材料的综述尚未见报道。另外, 无铅非线性介电储能陶瓷材料研究最近获得了飞速 发展，发现许多材料体系具有优良的储能特性，比 如, $\left(\mathrm{K}_{0.5} \mathrm{Na}_{0.5}\right) \mathrm{NbO}_{3}$ 基、 $\mathrm{BiFeO}_{3}$ 基和 $\mathrm{AgNbO}_{3}$ 基陶瓷。 结合本课题组的研究情况, 本文对无铅非线性介电 储能材料的研究进展进行系统评述, 最后指出不同 材料体系所面临的挑战和应对策略，以及下一步的 发展方向，以期为今后探寻高性能储能介质陶瓷材 料提供一定借鉴。

\section{1 基本原理}

电介质材料的储能密度 $(W)$ 是指电介质单位体 积内储存的能量, 单位为 $\mathrm{J} / \mathrm{cm}^{3}$, 可由式(1)表示 ${ }^{[29-30]}$ :

$$
W=\int_{0}^{D_{\max }} E \mathrm{~d} D
$$

其中 $E$ 为外加电场强度, $D$ 为电位移, $D_{\max }$ 表示电介 质在最大外加电场作用下的电位移。 $D$ 与 $E$ 又存在 如下关系(2):

$$
D=\varepsilon_{0} \varepsilon_{\mathrm{r}}
$$

其中 $\varepsilon_{\mathrm{r}}$ 为相对介电常数, $\varepsilon_{0}$ 为真空介电常数, 其值 为 $8.85 \times 10^{-12} \mathrm{~F} / \mathrm{m}$ 。

此外, 极化强度 $P$ 与 $D$ 满足式(3)所示关系,

$$
P=D-\varepsilon_{0} E=\varepsilon_{0}\left(\varepsilon_{\mathrm{r}}-1\right) E
$$

当 $\varepsilon_{\mathrm{r}}$ 较大时 $\left(\varepsilon_{\mathrm{r}} \gg 1\right), D$ 和 $P$ 值可以互换。因此, 材料 的 $W$ 又可以表示为式(4):

$$
W=\int_{0}^{P_{\max }} E \mathrm{~d} P
$$

其中 $P_{\max }$ 为电介质在 $E_{\mathrm{b}}$ 作用下的最大极化强度。 对于非线性介质材料, 在充电过程储存的能量并不 能完全释放。当 $E$ 从零增加到 $E_{\mathrm{b}}$, 电介质的 $P$ 达到 $P_{\max }$, 电容器充电, 储存的总能量为斜线面积和阴 影面积之和; 当 $E$ 从 $E_{\mathrm{b}}$ 减小到零, 材料的 $P$ 从 $P_{\text {max }}$ 减小到剩余极化强度 $\left(P_{\mathrm{r}}\right)$, 电容器放电, 如图 1(a) 所 示。由于 $P_{\mathrm{r}}$ 的存在, 电容器储存的能量并不能完全 释放, 可以释放出来的能量称为电介质材料的有效 储能密度 $\left(W_{\mathrm{rec}}\right)$, 即图 1(a)中的斜线面积, 可由式(5) 表示: 


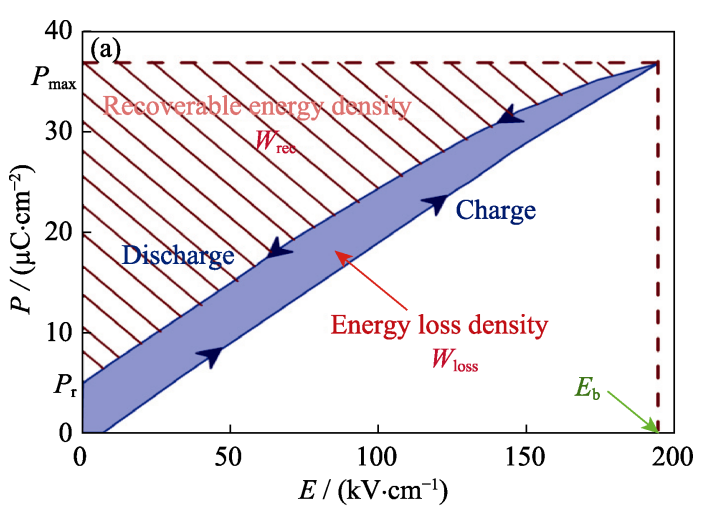

(b)

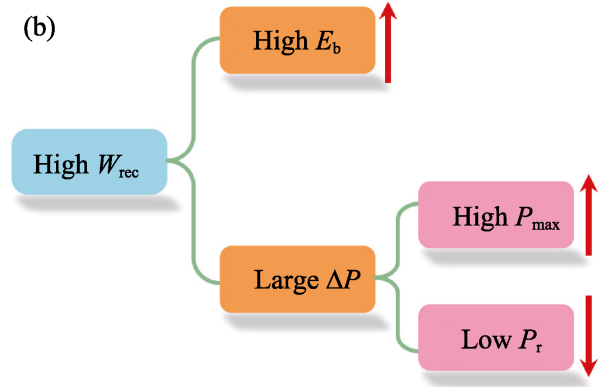

图 1 块体陶瓷储能密度计算及其影响因素示意图 ${ }^{[29-32]}$

Fig. 1 Schematic diagram of calculated storage density and its influence factors of bulk ceramics ${ }^{[29-32]}$

$$
W_{\text {rec }}=\int_{P_{\mathrm{r}}}^{P_{\max }} E \mathrm{~d} P
$$

不能释放的能量称为损失能量密度 $\left(W_{\text {loss }}\right)$, 图 1(a) 中的阴影面积。电介质材料的储能效率 $(\eta)$ 可用式(6) 表示:

$$
\eta=\frac{W_{\text {rec }}}{W_{\text {rec }}+W_{\text {loss }}}
$$

对于储能介质而言，理想的储能陶瓷应当具有 较高的 $W_{\mathrm{rec}}$ 和 $\eta$ 。根据上面的讨论, 要想获得较高 的 $W_{\mathrm{rec}}$ 和 $\eta$, 陶瓷介质材料必须同时满足两个条件: 极化差值大 $\left(\Delta P, \Delta P=P_{\text {max }}-P_{\mathrm{r}}\right)$ 和 $E_{\mathrm{b}}$ 高 ${ }^{[31-32]}$, 如图 1(b) 所示。也就是说, 陶瓷介质材料的 $P_{\max }$ 要尽可能的 大, $P_{\mathrm{r}}$ 要尽可能的小, $E_{\mathrm{b}}$ 要尽可能的高。

\section{2 材料体系}

根据电介质材料的 $P$ 随 $E$ 的变化规律, 储能介 质材料可分为线性和非线性两类 ${ }^{[1,4]}$ 。

对于线性材料, 它的 $\varepsilon$ 与 $E$ 无关, $D$ 与 $E$ 呈线性关 系。线性材料通常具有高的 $E_{\mathrm{b}}$, 但 $\varepsilon_{\mathrm{r}}$ 较低 $(\sim 100)^{[33-36]}$ 。 例如: Wei 等 ${ }^{[35}$ 报道了玻璃掺杂的 $\mathrm{TiO}_{2}$ 陶瓷, $E_{\mathrm{b}}$ 高达 $615.6 \mathrm{kV} / \mathrm{cm}$, 但 $\varepsilon_{\mathrm{r}}$ 较低 $\left(\varepsilon_{\mathrm{r}}=84\right)$, 这导致 $W_{\mathrm{rec}}$ 较低 $\left(W_{\mathrm{rec}}=1.41 \mathrm{~J} / \mathrm{cm}^{3}\right)$; Shay 等 ${ }^{[36]}$ 制备了 $\mathrm{MnO}_{2}$ 掺杂的 $0.8 \mathrm{CaTiO}_{3}-0.2 \mathrm{CaHfO}_{3}$ 单层陶瓷电容器, 在 $1300 \mathrm{kV} / \mathrm{cm}$ 外加电场作用下, $W_{\mathrm{rec}}$ 达到 $9.5 \mathrm{~J} / \mathrm{cm}^{3}$, 但 $\varepsilon_{\mathrm{r}}$ 却只有 $\sim 170$ 。文献[5]对该类介质材料的研究进展作了重点
讨论，本文不再赘述。

对于非线性材料, 它的 $\varepsilon$ 随 $E$ 的变化而变化, $D$ 与 $E$ 呈非线性关系。非线性储能介质主要包括弛豫 铁电材料和反铁电材料 ${ }^{[37-38]}$ 。其中弛豫铁电材料因 存在弥散相变而具有宽的平缓介电峰，因而温度稳 定性好。同时弛豫铁电材料具有瘦长型电滞回线, 在放电过程中释放出更多的能量, 具有较高的 $\eta$ 。因 此, 弛豫铁电陶瓷是一类非常重要的储能介质材 料。对于反铁电材料, 其重要特征是存在场致相变, 当外加 $E$ 较小时, 呈现反铁电相; 当外加 $E$ 高于反 铁电一铁电相变值时, 呈现铁电相, 这使得反铁电 材料同时具有铁电相的高 $P_{\max }$ 和反铁电相的低 $P_{\mathrm{r}}$ (几乎为零), 因而反铁电材料由于在理论上具有高 的 $W_{\mathrm{rec}}$ 和 $\eta$ 也得到广泛研究。

\section{1 无铅弛豫铁电材料}

\subsection{1 $\mathrm{BaTiO}_{3}$ (BT) 基材料}

$\mathrm{BT}$ 基陶瓷以其 $\varepsilon$ 高、 $\tan \delta$ 低和可靠性高等优 点在电子陶瓷领域获得了广泛研究和应用 ${ }^{[39-40]}$ 。近 年来，研究人员采用多种方法来提高 $\mathrm{BT}$ 基陶瓷的 $W_{\text {rec, }}$, 如掺杂改性 ${ }^{[41-42]}$, 表面包裹 ${ }^{[43-44]}$, 探索新的材 料体系 ${ }^{[45]}$, 制备陶瓷-聚合物复合材料 ${ }^{[46-47]}$ 等。在此, 本文主要对 $\mathrm{BT}-\mathrm{BiMeO}_{3}\left[\mathrm{Me}=\mathrm{Sc}^{3+} 、 \mathrm{Y}^{3+} 、 \mathrm{Yb}^{3+}\right.$ 和 $\left(\mathrm{Mg}_{1 / 2} \mathrm{Ti}_{1 / 2}\right)^{3+} 、\left(\mathrm{Zn}_{1 / 2} \mathrm{Ti}_{1 / 2}\right)^{3+} 、\left(\mathrm{Mg}_{2 / 3} \mathrm{Nb}_{1 / 3}\right)^{3+} 、\left(\mathrm{Zn}_{1 / 2} \mathrm{Zr}_{1 / 2}\right)^{3+} 、$ $\left(\mathrm{Li}_{1 / 2} \mathrm{Nb}_{1 / 2}\right)^{3+}$ ]类材料的研究进展进行评述。

为了同时提高 $\mathrm{BT}$ 基陶瓷的 $E_{\mathrm{b}}$ 和 $\Delta P$, 一些铋基 化合物被添加进 $\mathrm{BT}$, 形成了一系列 $\mathrm{BT}-\mathrm{BiMeO}_{3}$ 类 材料。该材料体系设计思路如图 2 所示, 主要基于 以下三个方面: (1) 引入铋基化合物能够降低烧结温 度，抑制晶粒生长，提高致密性进而提高其 $E_{\mathrm{b}}$; (2) 掺入铋基化合物能增强 $\mathrm{BT}$ 基陶瓷的弛豫特性，从而 降低其 $P_{\mathrm{r}}$; (3) 铋的 $6 \mathrm{p}$ 轨道与氧的 $2 \mathrm{p}$ 轨道杂化还可 以提高材料的 $P_{\max }$, 进而提高 $\Delta P$ 。2009 年, Ogihara 等 ${ }^{[48]}$ 采用固相烧结法制备了 $0.7 \mathrm{BaTiO}_{3}-0.3 \mathrm{BiScO}_{3}$ (0.7BT-0.3BS) 陶瓷, 在样品厚度为 $0.2 \mathrm{~mm}$ 的条件下, 其 $E_{\mathrm{b}}$ 达到 $225 \mathrm{kV} / \mathrm{cm}, W_{\mathrm{rec}}$ 达到 $2.3 \mathrm{~J} / \mathrm{cm}^{3}$, 在此基础 上, Ogihara 等 ${ }^{[48]}$ 通过流延法制备 $0.7 \mathrm{BT}-0.3 \mathrm{BS}$ 厚膜, 在膜厚度为 $15 \mu \mathrm{m}$ 的条件下, 其 $E_{\mathrm{b}}$ 达到 $730 \mathrm{kV} / \mathrm{cm}$, $W_{\text {rec }}$ 达到 $6.1 \mathrm{~J} / \mathrm{cm}^{3}$, 结果表明陶瓷介质材料的厚度 对 $E_{\mathrm{b}}$ 和 $W_{\mathrm{rec}}$ 具有重要影响。另外, 该材料可以在 $0 \sim 300^{\circ} \mathrm{C}$ 范围内保持良好的储能性能, 有望应用于高 温储能电容器。介电材料的 $W_{\mathrm{rec}}$, 很大程度上取决 于 $E_{\mathrm{b}}$ 。对于无机块体材料而言, 介电击穿的发生主 要是由于热击穿, 故陶瓷材料的厚度对其 $E_{\mathrm{b}}$ 有重要 影响, 进而影响 $W_{\mathrm{rec}}$ 的大小 ${ }^{[48-49]}$ 。因此, 今后在比较 不同介电块体材料的 $W_{\mathrm{rec}}$ 和 $E_{\mathrm{b}}$ 时, 还应给出样品的 尺寸以及 $E_{\mathrm{b}}$ 的测试方法。 


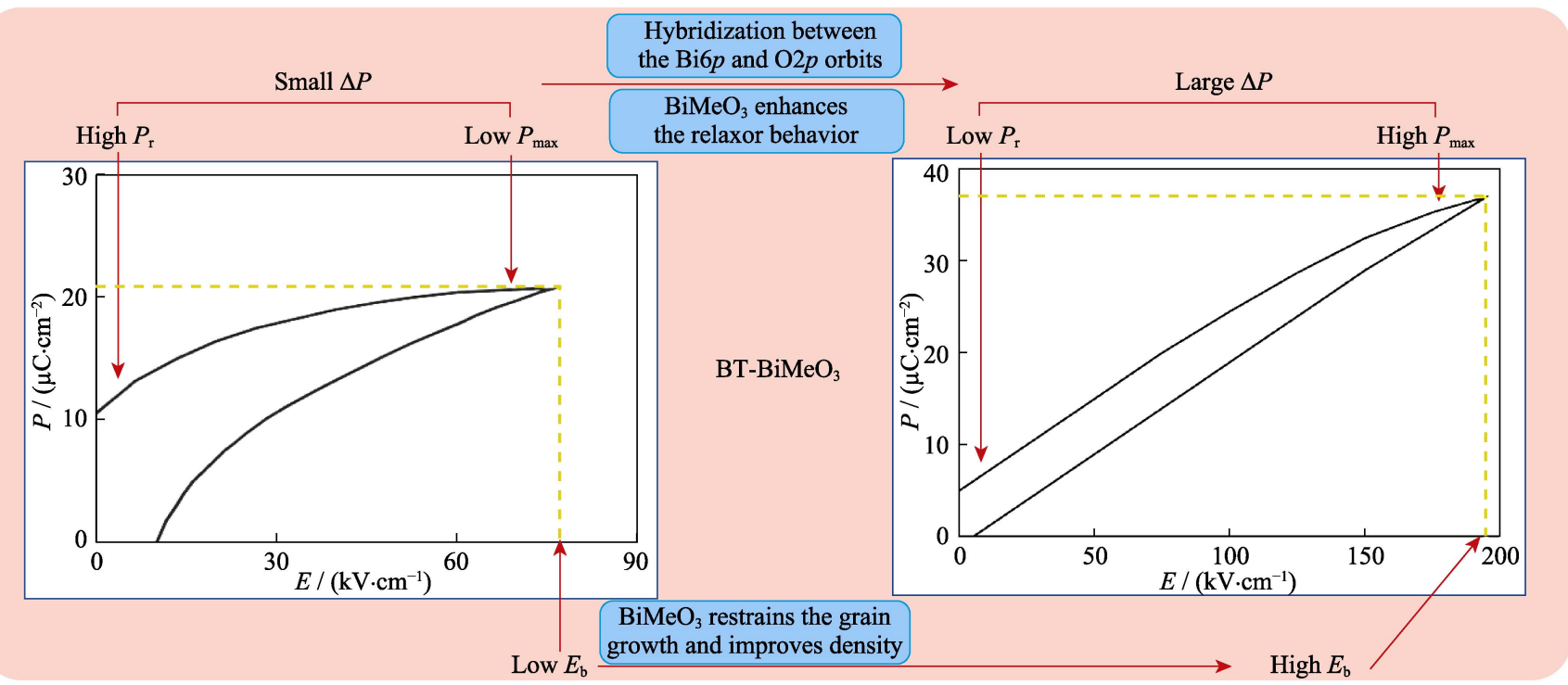

图 $2 \mathrm{BT}-\mathrm{BiMeO}_{3}$ 基材料体系的组分设计思路图 ${ }^{[51-55]}$

Fig. 2 Schematic diagram of composition design of $\mathrm{BT}-\mathrm{BiMeO}_{3}$ based material system ${ }^{[51-55]}$

随后 $\mathrm{Lim}$ 等 ${ }^{[50]}$ 用热压法制备了 $(1-x)\left(0.6 \mathrm{BaTiO}_{3}\right.$ $\left.0.4 \mathrm{BiScO}_{3}\right)-x\left(\mathrm{~K}_{1 / 2} \mathrm{Bi}_{1 / 2}\right) \mathrm{TiO}_{3}[(0.6 \mathrm{BT}-0.4 \mathrm{BS})-\mathrm{KBT}]$ 陶 瓷, 发现掺入 $\mathrm{KBT}$ 提高了材料的 $\varepsilon$, 增强了材料的 弛豫特性，同时采用热压法制备的材料致密性好、 气孔含量少, 从而具有高的 $E_{\mathrm{b}}$, 在 $0.17 \mathrm{~mm}$ 厚的 0.8 (0.6BT-0.4BS)-0.2KBT 样品的 $E_{\mathrm{b}}$ 为 $200 \mathrm{kV} / \mathrm{cm}, W_{\text {rec }}$ 达到 $2.7 \mathrm{~J} / \mathrm{cm}^{3}$, 当厚度降低到 $0.09 \mathrm{~mm}$ 时, $E_{\mathrm{b}}$ 提高到 $220 \mathrm{kV} / \mathrm{cm}, W_{\mathrm{rec}}$ 达到 $4.0 \mathrm{~J} / \mathrm{cm}^{3}$ 。随后, 一系列 $\mathrm{BT}-\mathrm{BiMeO}_{3}$ 基陶瓷被发展为新型储能介质材料。比 如，2014 年，Wang 等 ${ }^{[51]}$ 通过固相烧结法制备了 $(1-x) \mathrm{BaTiO}_{3}-\mathrm{xBi}\left(\mathrm{Mg}_{2 / 3} \mathrm{Nb}_{1 / 3}\right) \mathrm{O}_{3}$ (BT-BMN) 陶瓷, 研 究表明掺入 $\mathrm{BMN}$ 不仅可以降低材料的烧结温度, 而且能提高材料的致密性进而提高其 $E_{\mathrm{b}}$, 同时还可 以使材料表现出弛豫特性, 最终获得了瘦长型的电滞 回线, 并使 $0.3 \sim 0.5 \mathrm{~mm}$ 厚样品的 $E_{\mathrm{b}}$ 达到 $143.5 \mathrm{kV} / \mathrm{cm}$, $W_{\mathrm{rec}}$ 为 $1.13 \mathrm{~J} / \mathrm{cm}^{3}$, 此时 $\eta$ 为 $96 \%$ 。2015 年, Shen 等 ${ }^{[52]}$ 通过向 $\mathrm{BT}$ 中引入 $\mathrm{BiYbO}_{3}(\mathrm{BYO})$ 来降低材料的介电非 线性, 发现 BYO 不仅能提高 BT 的 $P_{\max }\left(\sim 17.5 \mu \mathrm{C} / \mathrm{cm}^{2}\right)$, 而且可以减小其 $P_{\mathrm{r}}\left(\sim 0.5 \mu \mathrm{C} / \mathrm{cm}^{2}\right)$, 进而获得瘦长型
的电滞回线, 0.91BT-0.09BYO 陶瓷在 $93 \mathrm{kV} / \mathrm{cm}$ 的 外加电场作用下, $W_{\mathrm{rec}}$ 达到 $0.71 \mathrm{~J} / \mathrm{cm}^{3}, \eta$ 为 $87 \%$ 。

最近, 西安交通大学的 $\mathrm{Li}$ 等 ${ }^{[4]}$ 和 Yuan 等 ${ }^{[5]}$ 分别 向 $\mathrm{BT}$ 中引入 $\mathrm{Bi}\left(\mathrm{Li}_{1 / 2} \mathrm{Nb}_{1 / 2}\right) \mathrm{O}_{3}(\mathrm{BLN})$ 和 $\mathrm{Bi}\left(\mathrm{Zn}_{1 / 2} \mathrm{Zr}_{1 / 2}\right) \mathrm{O}_{3}$ (BZZ)来增加样品的弛豫特性并提高 $E_{\mathrm{b}}$, 最终制备 出具有高 $W_{\mathrm{rec}}$ 和优良温度稳定性的弛豫铁电陶瓷材 料, 并研究了它们的快速放电特性。0.88BT$0.12 \mathrm{BLN}$ 陶瓷在 $270 \mathrm{kV} / \mathrm{cm}$ 的外加电场作用下, $W_{\mathrm{rec}}$ 达到 $2.032 \mathrm{~J} / \mathrm{cm}^{3}$, 此时 $\eta$ 为 $88 \%$, 快速放电时间 $\left(t_{0.9}<\right.$ $0.5 \mu \mathrm{s})$, 并可以在 $20 \sim 120^{\circ} \mathrm{C}$ 范围内保持良好的储能 性能 ${ }^{[54]}$ 。0.9BT-0.1BZZ 陶瓷厚度为 $0.2 \mathrm{~mm}$ 时, 其最 大 $E_{\mathrm{b}}$ 达到 $264 \mathrm{kV} / \mathrm{cm}, W_{\mathrm{rec}}$ 为 $2.46 \mathrm{~J} / \mathrm{cm}^{3}, t_{0.9}$ 为 $16.48 \mu \mathrm{s}$, 并可以在 $30 \sim 150^{\circ} \mathrm{C}$ 范围内保持良好的储能性能 ${ }^{[55]}$ 。 这也证实了高 $W_{\mathrm{rec}}$ 的介电材料不一定放电速度快, 而介电材料在实际应用中不仅需要高 $W_{\mathrm{rec}}$ 也需要小 $t_{0.9}$, 因此研究还应关注材料的快速放电性能, 此外 由脉冲放电回路和由电滞回线计算出的储能密度存 在一定的差距, 其中的原因也有待研究 ${ }^{[54-56]}$ 。表 1 给了一些具有代表性的 BT 基陶瓷的储能性能。

表 1 一些代表性的 BT 基陶瓷的储能性能

Table 1 Energy storage performance of some representative BT-based ceramics

\begin{tabular}{|c|c|c|c|c|c|}
\hline Materials & $E_{\mathrm{b}} /\left(\mathrm{kV} \cdot \mathrm{cm}^{-1}\right)$ & $\Delta P /\left(\mu \mathrm{C} \cdot \mathrm{cm}^{-2}\right)$ & $W_{\mathrm{rec}} /\left(\mathrm{J} \cdot \mathrm{cm}^{-3}\right)$ & $\eta / \%$ & Ref. \\
\hline $0.7 \mathrm{BaTiO}_{3}-0.3 \mathrm{BiScO}_{3}$ & 225 & 25 & 2.3 & - & [48] \\
\hline $0.8\left(0.6 \mathrm{BaTiO}_{3}-0.4 \mathrm{BiScO}_{3}\right)-0.2\left(\mathrm{~K}_{1 / 2} \mathrm{Bi}_{1 / 2}\right) \mathrm{TiO}_{3}$ & 220 & $\sim 33.5$ & 4.0 & - & {$[50]$} \\
\hline $0.91 \mathrm{BaTiO}_{3}-0.09 \mathrm{BiYbO}_{3}$ & 93 & $\sim 17$ & 0.71 & $\sim 87$ & [52] \\
\hline $0.9 \mathrm{BaTiO}_{3}-0.1 \mathrm{Bi}\left(\mathrm{Mg}_{2 / 3} \mathrm{Nb}_{1 / 3}\right) \mathrm{O}_{3}$ & 143.5 & $\sim 15$ & 1.13 & $\sim 96$ & [51] \\
\hline $0.88 \mathrm{BaTiO}_{3}-0.12 \mathrm{Bi}\left(\mathrm{Mg}_{1 / 2} \mathrm{Ti}_{1 / 2}\right) \mathrm{O}_{3}$ & 224 & $\sim 19$ & 1.81 & $\sim 88$ & [23] \\
\hline $0.85 \mathrm{BaTiO}_{3}-0.15 \mathrm{Bi}\left(\mathrm{Zn}_{2 / 3} \mathrm{Nb}_{1 / 3}\right) \mathrm{O}_{3}$ & 131 & 7.9 & 0.79 & 93.5 & [53] \\
\hline $0.88 \mathrm{BaTiO}_{3}-0.12 \mathrm{Bi}\left(\mathrm{Li}_{1 / 2} \mathrm{Nb}_{1 / 2}\right) \mathrm{O}_{3}$ & 270 & 13.35 & 2.032 & 88 & [54] \\
\hline $0.9 \mathrm{BaTiO}_{3}-0.1 \mathrm{Bi}\left(\mathrm{Zn}_{1 / 2} \mathrm{Zr}_{1 / 2}\right) \mathrm{O}_{3}$ & 264 & 22.1 & 2.46 & - & {$[55]$} \\
\hline
\end{tabular}




\subsection{2 $\mathrm{BiFeO}_{3}(\mathrm{BF})$ 基材料}

$\mathrm{BF}$ 基陶瓷以其高的 $P_{\max }$ 和高的 $T_{\mathrm{c}}\left(825^{\circ} \mathrm{C}\right)$ 而被 认为是制备耐高温储能电容器的理想材料 ${ }^{[56-57]}$ 。但 是纯 $\mathrm{BF}$ 陶瓷不仅 $E_{\mathrm{c}}$ 和 $P_{\mathrm{r}}$ 较大, 而且漏电流较大, 导致其 $E_{\mathrm{b}}$ 较低，这限制了它在储能领域的应用 ${ }^{[58-59]}$ 。 目前提高该材料体系储能密度的设计思路有两个方 向：一方面，通过 $\mathrm{A} 、 \mathrm{~B}$ 位掺杂来产生尺寸小、动力 学性能好的极性纳米微区(PNRs), 增强其弛豫特性, 进而减小其 $P_{\mathrm{r}}$; 另一方面, 通过制备缺陷少的薄膜 或利用 $\mathrm{MnO}_{2}$ 填补氧空位来抑制漏电流，提高其 $E_{\mathrm{b}}$ 。 最终获得了高储能密度的电介质材料(如图 3 所示)。

目前, $\mathrm{BF}$ 基材料体系主要有两类: $\mathrm{BiFeO}_{3}-\mathrm{BaTiO}_{3}$ (BF-BT) 和 $\mathrm{BiFeO}_{3}-\mathrm{SrTiO}_{3}(\mathrm{BF}-\mathrm{ST})$ 。

BF-BT 基陶瓷由于 $P_{\mathrm{r}}$ 较高和明显的迟滞效应， 所以 $W_{\mathrm{rec}}$ 较低。研究人员主要是在保持其高 $P_{\max }$ 的 基础上，通过降低 $P_{\mathrm{r}}$ 来获得大的 $\Delta P$, 从而提高 $W_{\text {rec }}$ 。如: Wang 等 ${ }^{[60]}$ 通过向 BF-BT 陶瓷中掺入 $\mathrm{Nb}_{2} \mathrm{O}_{5}$ 来降低其 $P_{\mathrm{r}}$, 结果表明掺入 $\mathrm{Nb}_{2} \mathrm{O}_{5}$ 使材料在保持较 高 $P_{\max }\left(25.21 \mu \mathrm{C} / \mathrm{cm}^{2}\right)$ 的基础上可以将 $P_{\mathrm{r}}$ 降低到 $5.33 \mu \mathrm{C} / \mathrm{cm}^{2}$, 而且 $\mathrm{Nb}_{2} \mathrm{O}_{5}$ 的掺入还可以减小陶瓷晶 粒尺寸, 进而提高材料的 $E_{\mathrm{b}}$, 最终在 $90 \mathrm{kV} / \mathrm{cm}$ 的外 加电场下获得 $0.71 \mathrm{~J} / \mathrm{cm}^{3}$ 的 $W_{\text {rec }}$ ZZheng 等 ${ }^{[61-62]}$ 通过
向 BF-BT 陶瓷中引入新组元 $\mathrm{La}\left(\mathrm{Mg}_{1 / 2} \mathrm{Ti}_{1 / 2}\right) \mathrm{O}_{3}$ 来增加 弛豫特性，降低 $P_{\mathrm{r}}$, 提高 $\Delta P$, 厚度为 $0.5 \mathrm{~mm}$ 的样品的 最大 $E_{\mathrm{b}}$ 达到 $130 \mathrm{kV} / \mathrm{cm}, P_{\text {max }}$ 高达 $37.5 \mu \mathrm{C} / \mathrm{cm}^{2}, P_{\mathrm{r}}$ 降 低到 $4.2 \mu \mathrm{C} / \mathrm{cm}^{2}, W_{\text {rec }}$ 为 $1.66 \mathrm{~J} / \mathrm{cm}^{3}$, 此时 $\eta$ 为 $75 \%$, 而且该材料可以在 $25 \sim 180^{\circ} \mathrm{C}$ 范围内保持良好储能 性能。

BF-ST 基陶瓷的主要特点是 $\tan \delta$ 小、温度稳定 性好。Correia 等 ${ }^{[63]}$ 采用脉冲激光沉积技术制备了厚 度为 $400 \mathrm{~nm}$ 的 $0.4 \mathrm{BF}-0.6 \mathrm{ST}$ 薄膜, 在 $972 \mathrm{kV} / \mathrm{cm}$ 电 场下, $P_{\max }$ 高达 $52 \mu \mathrm{C} / \mathrm{cm}^{2}, P_{\mathrm{r}}$ 降低到 $3 \mu \mathrm{C} / \mathrm{cm}^{2}, W_{\mathrm{rec}}$ 达到 $18.6 \mathrm{~J} / \mathrm{cm}^{3}, \eta$ 大于 $85 \%$ 。之后, Correia 等 ${ }^{[64]}$ 还 利用丝网印刷法制备了 $0.4 \mathrm{BF}-0.6 \mathrm{ST}$ 厚膜，在厚度 为 $11 \mu \mathrm{m}$ 的条件下，其 $E_{\mathrm{b}}$ 达到 $420 \mathrm{kV} / \mathrm{cm}, W_{\text {rec }}$ 达到 $6 \mathrm{~J} / \mathrm{cm}^{3}$, 并且能够在 $20 \sim 200^{\circ} \mathrm{C}$ 范围内保持较好储能 性能。这些结果证实，在材料组分相同的情况下，薄 膜比厚膜具有更高的 $E_{\mathrm{b}}$ 和 $W_{\mathrm{rec}}$ 。近年来, 为了进一 步提高 BF-ST 基陶瓷的储能密度, Pan 等 ${ }^{[65]}$ 利用脉冲 激光沉积法制备了厚度为 $500 \mathrm{~nm}$ 的 $0.4 \mathrm{BF}-0.6 \mathrm{ST}+$ $0.5 \% \mathrm{MnO}_{2}$ 薄膜，使其 $E_{\mathrm{b}}$ 达到 $3600 \mathrm{kV} / \mathrm{cm}, W_{\mathrm{rec}}$ 达 到 $51 \mathrm{~J} / \mathrm{cm}^{3}$, 并且该材料具有很好的抗疲劳特性 ( $2 \times 10^{7}$ 次), 其优异性能可归于以下三个方面: (1) 如 式(7)、(8)所示, 通过引入 $\mathrm{MnO}_{2}$ 可以填补其中的氧

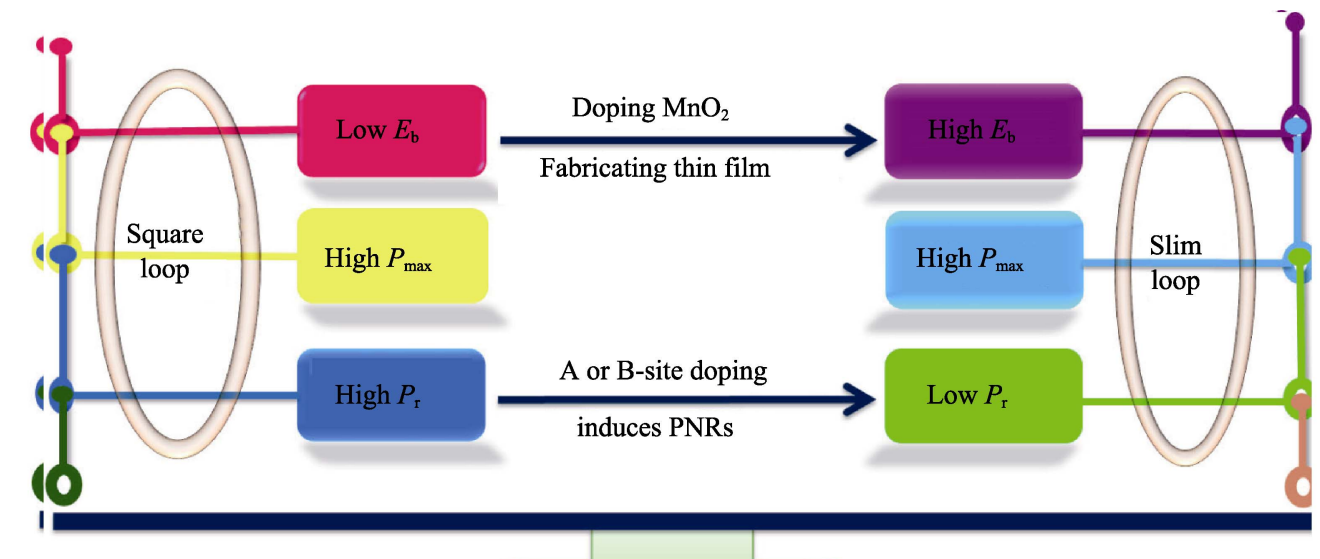

The hysteresis loop transforms from square to slim
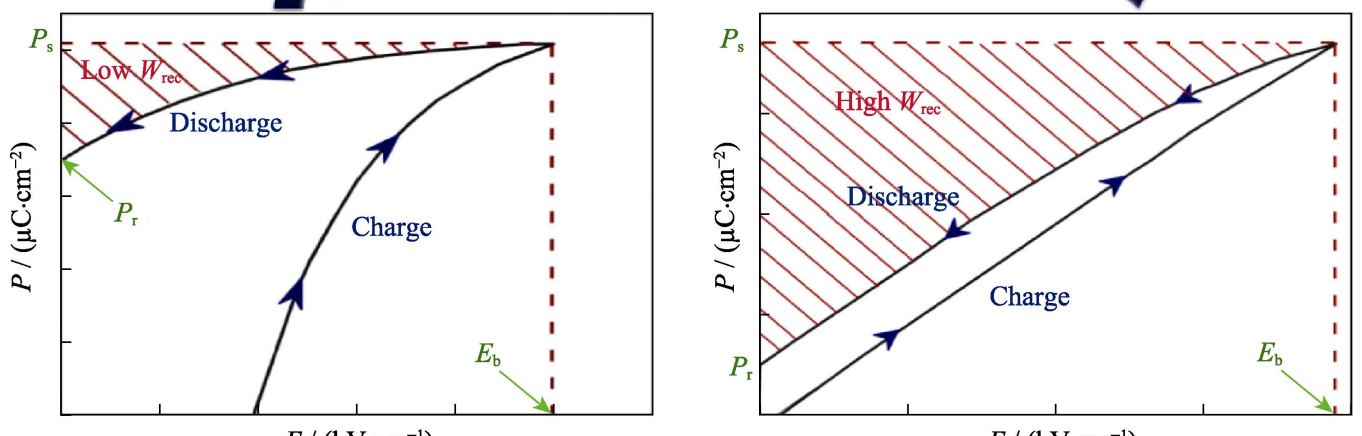

$E /\left(\mathrm{kV} \cdot \mathrm{cm}^{-1}\right)$

图 $3 \mathrm{BF}$ 基材料体系的组分设计思路图 ${ }^{[60-65]}$

Fig. 3 Schematic diagram of composition design of BF based material system ${ }^{[60-65]}$ 
空位, 抑制漏电流, 有利于获得高 $E_{\mathrm{b}}$; (2) 用脉冲激 光沉积技术制备了厚度薄, 质量高的薄膜(致密高、 定向高、外延质量好、晶格不匹配低), 这都有利于 获得高 $E_{\mathrm{b}}$; (3) 该材料具有强的弛豫特性, 有利于获 得较大的 $\Delta P$ 和优良的抗疲劳特性。最终使得该材 料具有优异的储能特性。表 2 概括了一些 $\mathrm{BF}$ 基陶 瓷材料的储能性能。

$$
\begin{gathered}
2 \mathrm{Fe}_{\mathrm{Fe}}+\mathrm{O}_{\mathrm{o}} \rightarrow 2 \mathrm{Fe}_{\mathrm{Fe}}^{\prime}+\mathrm{V}_{\mathrm{o}}^{*}+\frac{1}{2} \mathrm{O}_{2} \\
2 \mathrm{Mn}_{\mathrm{Fe}}^{\prime}+\mathrm{V}_{\mathrm{o}}^{*}+\frac{1}{2} \mathrm{O}_{2} \rightarrow 2 \mathrm{Mn}_{\mathrm{Fe}}+\mathrm{O}_{\mathrm{o}}
\end{gathered}
$$

\subsection{3 $\left(\mathrm{K}_{0.5} \mathrm{Na}_{0.5}\right) \mathrm{NbO}_{3}(\mathrm{KNN})$ 基材料}

近 10 多年来, $\mathrm{KNN}$ 基陶瓷以其优良的压电性和 环境友好性而备受关注。研究内容主要集中在能量 转换上, 包括压电效应 ${ }^{[66-67]}$ 、电光效应 ${ }^{[68-69]}$ 、电卡 效应 ${ }^{[70-71]}$, 而对其电能存储特性的研究却少有报 道。纯 $\mathrm{KNN}$ 陶瓷是一种正常的铁电体, 不仅 $E_{\mathrm{b}}$ 很 低 $(40 \mathrm{kV} / \mathrm{cm})$, 而且由于 $P_{\mathrm{r}}$ 较大, 致使 $\Delta P$ 也很小 $\left(<5 \mu \mathrm{C} / \mathrm{cm}^{2}\right)$, 这就导致其不能用作储能介质。最近, 本课题组利用 $\mathrm{KNN}$ 基陶瓷独特的亚微米晶粒, 发 展了一系列具有高 $E_{\mathrm{b}}$ 和 $W_{\mathrm{rec}}$ 的 $\mathrm{KNN}$ 基陶瓷材料体 系, 下面从材料体系的设计入手, 概括 $\mathrm{KNN}$ 基储能 陶瓷的组分设计思路。

对于块体陶瓷而言, 其 $E_{\mathrm{b}}$ 主要与材料本身的气 孔、晶粒大小、致密性等因素有关, 其中晶粒大小 是一个重要的因素 ${ }^{[72-73]}$, 通过减小晶粒尺寸可以增 加陶瓷致密性和减少气孔含量, 进而达到提高 $E_{\mathrm{b}}$ 的 效果。研究证实介电陶瓷的 $E_{\mathrm{b}}$ 与晶粒尺寸之间的关 系如式(9)所示 ${ }^{[74]}$.

$$
E_{\mathrm{b}} \propto \frac{1}{\sqrt{G}}
$$

这里, $G$ 代表陶瓷材料的晶粒大小。从式(9)可以看 出, 晶粒尺寸越小, $E_{\mathrm{b}}$ 越大。

此外, 由图 4 所示的介电陶瓷的 $E_{\mathrm{b}}$ 与晶粒大小 的关系图同样可得: 对于同一个组分, $E_{\mathrm{b}}$ 随晶粒尺 寸的减小而增大, 并且当晶粒尺寸降到亚微米时, $E_{\mathrm{b}}$ 将会成倍地提高 ${ }^{[75-78]}$ 。这也是采用热压烧结法或 火花等离子烧结法制备的陶瓷具有高 $E_{\mathrm{b}}$ 的重要原
因之一[79-80]。

在 $\mathrm{KNN}$ 基陶瓷的众多研究成果中, 有一个独 特的现象值得注意: 添加一些化合物可以使采用无 压烧结法制备的 $\mathrm{KNN}$ 基陶瓷的晶粒尺寸减小到亚 微米尺度, 而无需借助热压或放电等离子烧结技术。 Koruza 等 ${ }^{[81]}$ 报道了 $0.85 \mathrm{~K}_{0.5} \mathrm{Na}_{0.5} \mathrm{NbO}_{3}-0.15 \mathrm{SrTiO}_{3}$ $(0.85 \mathrm{KNN}-0.15 \mathrm{ST})$ 陶瓷的平均晶粒大小为 $0.4 \mu \mathrm{m}$ 。 后来, $\mathrm{Liu}$ 等 ${ }^{[82]}$ 又证实 $0.82 \mathrm{KNN}-0.18 \mathrm{ST}$ 陶瓷同样具 有亚微米级晶粒 $(0.2 \sim 0.4 \mu \mathrm{m})$ 。本课题组 ${ }^{[83]}$ 在前期研 究 $\mathrm{KNN}$ 基陶瓷的电光效应时也发现 $0.95\left(\mathrm{~K}_{0.5} \mathrm{Na}_{0.5}\right)$ $\mathrm{NbO}_{3}-0.05 \mathrm{Ba}\left(\mathrm{Sc}_{0.5} \mathrm{Nb}_{0.5}\right) \mathrm{O}_{3}$ 陶瓷具有亚微米晶粒 $(0.2 \sim 0.3 \mu \mathrm{m})$ 。为了研究具有亚微米级晶粒的 $\mathrm{KNN}$ 基陶瓷的 $E_{\mathrm{b}}$ 和储能特性, 本课题组设计了 $(1-x)$ $\mathrm{K}_{0.5} \mathrm{Na}_{0.5} \mathrm{NbO}_{3}-x \mathrm{Sr}\left(\mathrm{Sc}_{0.5} \mathrm{Nb}_{0.5}\right) \mathrm{O}_{3}[(1-x) \mathrm{KNN}-x \mathrm{SSN}]^{[84]}$ 和 $(1-x) \mathrm{KNN}-x \mathrm{ST}^{[85]}$ 两个组分, 期望 $\mathrm{SSN}$ 或 $\mathrm{ST}$ 的掺 入能够将 $\mathrm{KNN}$ 陶瓷的晶粒尺寸降到亚微米尺度, 从而提高其 $E_{\mathrm{b}}$, 获得高的 $W_{\mathrm{rec}}$, 设计思路如图 5 所 示。结果表明: $0.8 \mathrm{KNN}-0.2 \mathrm{SSN}$ 陶瓷的平均晶粒尺 寸为 $0.5 \mu \mathrm{m}$, 当样品厚度为 $0.2 \mathrm{~mm}$ 时, $0.8 \mathrm{KNN}$ $0.2 \mathrm{SSN}$ 陶瓷的 $E_{\mathrm{b}}$ 由原来纯 $\mathrm{KNN}$ 陶瓷的 $40 \mathrm{kV} / \mathrm{cm}$ 提高到 $295 \mathrm{kV} / \mathrm{cm}, W_{\mathrm{rec}}$ 达到 $2.02 \mathrm{~J} / \mathrm{cm}^{3}$ 。值得关注的 是, $0.8 \mathrm{KNN}-0.2 \mathrm{SSN}$ 陶瓷还具有较高的透光性, 这 些结果证实 $0.8 \mathrm{KNN}-0.2 \mathrm{SSN}$ 陶瓷是一个多功能陶 瓷材料。而 $\mathrm{ST}$ 的掺入还可以使 $\mathrm{KNN}$ 陶瓷的平均 晶粒尺寸减小, $0.85 \mathrm{KNN}-0.15 \mathrm{ST}$ 陶瓷的平均晶粒大 小为 $0.3 \mu \mathrm{m}$, 当样品厚度为 $0.2 \mathrm{~mm}$ 时, 其 $E_{\mathrm{b}}$ 达到 $400 \mathrm{kV} / \mathrm{cm}, W_{\text {rec }}$ 达到 $4.03 \mathrm{~J} / \mathrm{cm}^{3}$ 。

要想获得高 $W_{\mathrm{rec}}$, 除了提高 $E_{\mathrm{b}}$ 之外, 必须尽可 能地增大 $\Delta P$ 。要想获得大的 $\Delta P$ 需要材料同时具有 高 $P_{\max }$ 和低 $P_{\mathrm{r}}$, 提高 $P_{\max }$ 可以通过提高 $\mathrm{A}$ 位离子的 极性来实现，而降低材料的 $P_{\mathrm{r}}$ 可以通过增加材料的 弛豫特性获得。近年来,一些 $\mathrm{BiMeO}_{3}$ 化合物因可以 提高介质材料的储能特性而得到了广泛研究 ${ }^{[50-55]}$ 。添 加 $\mathrm{BiMeO}_{3}$ 化合物主要有三个优点: (1) 处于钻钛矿 结构中 $\mathrm{A}$ 位的 $\mathrm{Bi}^{3+}$ 与 $\mathrm{Pb}^{2+}$ 具有类似的电子结构, 添 加 $\mathrm{Bi}^{3+}$ 可以提高 $\mathrm{A}$ 位离子的极性进而提高材料 $P_{\text {max }}$; (2) 添加 $\mathrm{BiMeO}_{3}$ 固溶物可以增加材料的弛豫特性, 降低其 $P_{\mathrm{r}} ;(3)$ 添加 $\mathrm{BiMeO}_{3}$ 固溶物能够抑制 $\mathrm{KNN}$

表 2 一些 BF 基陶瓷的储能性能

Table 2 Energy storage properties of some BF-based ceramics

\begin{tabular}{cccccccc}
\hline Materials & Compositions & Marterial forms & $E_{\mathrm{b}} /\left(\mathrm{kV} \cdot \mathrm{cm}^{-1}\right)$ & $\Delta P /\left(\mu \mathrm{C} \cdot \mathrm{cm}^{-2}\right)$ & $W_{\text {red }} /\left(\mathrm{J} \cdot \mathrm{cm}^{-3}\right)$ & $\eta / \%$ & Ref. \\
\hline BF-BT & $0.7(0.65 \mathrm{BF}-0.35 \mathrm{BT})-0.3 \mathrm{Nb}_{2} \mathrm{O}_{5}$ & Bulk & 90 & 19.68 & 0.71 & - & {$[60]$} \\
& $0.61 \mathrm{BF}-0.33 \mathrm{BT}-0.06 \mathrm{La}\left(\mathrm{Mg}_{1 / 2} \mathrm{Ti}_{1 / 2}\right) \mathrm{O}_{3}$ & Bulk & 130 & 33.3 & 1.66 & 82 & {$[61]$} \\
& $0.61 \mathrm{BF}-0.33 \mathrm{BT}-0.06 \mathrm{Ba}\left(\mathrm{Mg}_{1 / 3} \mathrm{Nb}_{2 / 3}\right) \mathrm{O}_{3}$ & Bulk & 125 & 32.3 & 1.56 & 75 & {$[62]$} \\
\multirow{2}{*}{ BF-ST } & $0.4 \mathrm{BF}-0.6 \mathrm{ST}$ & Thick film & 420 & - & 6 & - & {$[64]$} \\
& $0.4 \mathrm{BF}-0.6 \mathrm{ST}+0.5 \% \mathrm{MnO}_{2}$ & Thin film & 3600 & $\sim 38$ & $\sim 51$ & 64 & {$[65]$} \\
\hline
\end{tabular}




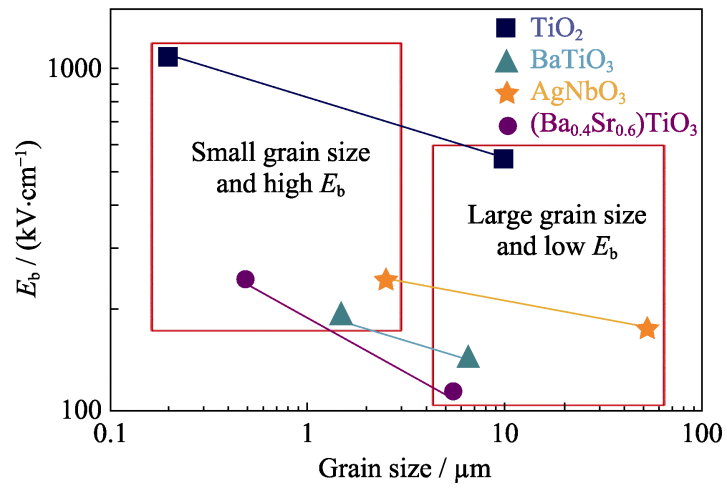

图 4 一些典型介电陶瓷的 $E_{\mathrm{b}}$ 与晶粒大小的关系图 ${ }^{[75-78]}$ Fig. $4 E_{\mathrm{b}}$ and grain size of some typical dielectric ceraics ${ }^{[75-78]}$

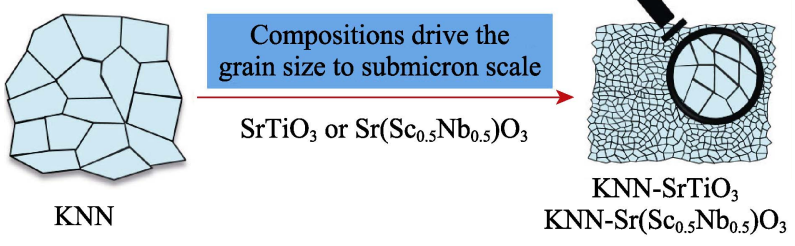

图 $5 \mathrm{KNN}-\mathrm{ST}, \mathrm{KNN}-\mathrm{SSN}$ 材料的组分设计思路图 ${ }^{[84-85]}$

Fig. 5 Schematic diagram of composition design of KNN-ST and KNN-SSN material system ${ }^{[84-85]}$

基陶瓷晶粒的生长, 使陶瓷晶粒大小降低到亚微米 级, 从而获得较高的 $E_{\mathrm{b}}$ 。基于以上分析,设计了 $(1-x) \mathrm{K}_{0.5} \mathrm{Na}_{0.5} \mathrm{NbO}_{3}-x \mathrm{Bi}\left(\mathrm{Mg}_{2 / 3} \mathrm{Nb}_{1 / 3}\right) \mathrm{O}_{3}(\mathrm{KNN}-\mathrm{BMN})$ 陶 瓷组份 ${ }^{[86]}$, 结果表明当厚度为 $0.2 \mathrm{~mm}$ 时, $0.90 \mathrm{KNN}$ $0.10 \mathrm{BMN}$ 陶瓷不仅具有较大的 $\Delta P\left(\Delta P=33 \mu \mathrm{C} / \mathrm{cm}^{2}\right)$, 而且具有较高的 $E_{\mathrm{b}}(300 \mathrm{kV} / \mathrm{cm}), W_{\mathrm{rec}}$ 达到 $4.08 \mathrm{~J} / \mathrm{cm}^{3}$, 是其他无铅块体陶瓷 $W_{\mathrm{rec}}$ 的 2 3 倍。

除了晶粒尺寸, 气孔对陶瓷材料的 $E_{\mathrm{b}}$ 也有重要 影响, 气孔越少, 致密度越高, $E_{\mathrm{b}}$ 越大。图 6 为传统 固相烧结法和过渡液相烧结法的对比图, 由图可以 看出, 过渡液相烧结不仅可以降低材料的烧结温度, 而且可以减少材料中的气孔含量, 增强陶瓷的致密 性, 进而提高其 $E_{\mathrm{b}}$, 最终提高材料的储能特性 ${ }^{[87-89]}$ 。

目前, 过渡液相烧结的烧结助剂主要有两类: 一类是氧化物 $(\mathrm{CuO} 、 \mathrm{ZnO})$, 在烧结过程中这些氧化

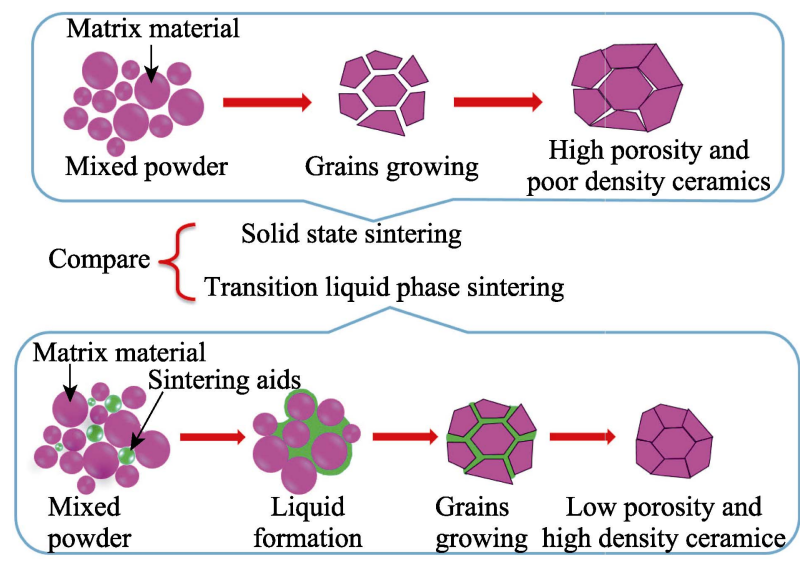

图 6 传统固相烧结和过渡液相烧结的对比图 ${ }^{[90-91]}$

Fig. 6 Comparison of solid state sintering and transition liquid phase sintering ${ }^{[90-91]}$

物依靠自身或者与基体材料一起产生的少量液相, 促进烧结进行，减少气孔的形成; 另一类是本身熔 点就低的玻璃粉。但是, 玻璃粉虽然可以在一定程 度上增加陶瓷的致密性进而提高其 $E_{\mathrm{b}}$, 但同时也会使 陶瓷的介电性能恶化。本课题组研究证实加入 $\mathrm{ZnO}$ 可以增加陶瓷的致密性进而提高材料的 $E_{\mathrm{b}}$, 最终将 $0.8 \mathrm{KNN}-0.2 \mathrm{SSN}$ 陶瓷的 $E_{\mathrm{b}}$ 由原来的 $295 \mathrm{kV} / \mathrm{cm}$ 提高

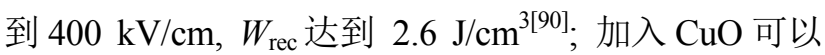
显著降低陶瓷的烧结温度 $\left(\sim 940^{\circ} \mathrm{C}\right)$, 这使其在多层 电容器制备方面具有很大优势, 在样品厚度为 $(0.2 \pm$ $0.05) \mathrm{mm}$ 时, $E_{\mathrm{b}}$ 达到 $400 \mathrm{kV} / \mathrm{cm}, W_{\mathrm{rec}}$ 为 $4.02 \mathrm{~J} / \mathrm{cm}^{3[91]}$ 。 过渡液相烧结的关键是精确控制氧化物的添加量, 添加量过大, 将产生大量液相, 促进晶粒生长, 造 成 $\mathrm{KNN}$ 基陶瓷晶粒的异常长大，降低 $E_{\mathrm{b}}$; 添加量过 小, 起不到减少气孔含量的作用。因此, 精确控制烧 结助剂添加量是确保过渡液相烧结质量的关键。表 3 归纳了 $\mathrm{KNN}$ 基陶瓷材料的储能性能。

\section{2 无铅反铁电材料}

\subsection{1 $\mathrm{AgNbO}_{3}(\mathrm{AN})$ 基陶瓷}

近几年, $\mathrm{AN}$ 陶瓷作为一种有望替代含铅陶瓷的 候选材料得到了广泛研究。AN 是一种具有中心对 称的 $\mathrm{Pbcm}$ 型空间群的 $\mathrm{ABO}_{3}$ 型钙钛矿结构材料, 在 室温和低电场条件下表现为弱铁电相 $(\mathrm{FE})$, 当温度

表 $3 \mathrm{KNN}$ 基陶瓷的储能性能

Table 3 Energy storage properties of KNN-based ceramics

\begin{tabular}{|c|c|c|c|c|c|c|}
\hline Materials systems & Grain size $/ \mu \mathrm{m}$ & $E_{\mathrm{b}} /\left(\mathrm{kV} \cdot \mathrm{cm}^{-1}\right)$ & $\Delta P /\left(\mu \mathrm{C} \cdot \mathrm{cm}^{-2}\right)$ & $W_{\mathrm{rec}} /\left(\mathrm{J} \cdot \mathrm{cm}^{-3}\right)$ & $\eta / \%$ & Ref. \\
\hline $\mathrm{KNN}$ & 2.26 & 40 & 5 & - & - & [85] \\
\hline $0.8 \mathrm{KNN}-0.2 \mathrm{SSN}$ & 0.5 & 295 & 13.8 & 2.02 & 81.4 & [84] \\
\hline $0.85 \mathrm{KNN}-0.15 \mathrm{ST}$ & 0.3 & 400 & 25.8 & 4.03 & 52 & [85] \\
\hline $0.90 \mathrm{KNN}-0.10 \mathrm{BMN}$ & 0.31 & 300 & 33.0 & 4.08 & 62.7 & [86] \\
\hline $0.8 \mathrm{KNN}-0.2 \mathrm{SSN}+0.5 \% \mathrm{ZnO}$ & 0.45 & 400 & 14.7 & 2.6 & 73.2 & [90] \\
\hline $0.90 \mathrm{KNN}-0.10 \mathrm{BMN}+1.0 \% \mathrm{CuO}$ & 0.49 & 400 & 26 & 4.02 & 57.3 & [91] \\
\hline
\end{tabular}


升高或外加电场强度增加时表现为反铁电相 $(\mathrm{AFE})^{[92-93]}$ 。最近, AN 基陶瓷开始用于储能领域, 这 主要是因为其本身具有中等的 $E_{\mathrm{b}}(\sim 150 \mathrm{kV} / \mathrm{cm})$, 和 较高的 $P_{\max }$, 从而 $W_{\mathrm{rec}}$ 较高。目前, 提高该材料体 系储能密度的设计思路如图 7 所示, 在保持其高 $P_{\max }$ 的基础上, 通过进一步提高其反铁电一铁电相 变电场 $E_{\mathrm{F}}$ 、铁电一反铁电相变电场 $E_{\mathrm{A}}$ 以及 $E_{\mathrm{b}}$ 并减小 $\Delta E\left(\Delta E=E_{\mathrm{F}}-E_{\mathrm{A}}\right)$ 来提高其 $W_{\mathrm{rec}}$ 。如: Tian 等 ${ }^{[94]}$ 通过 传统的固相烧结法制备了纯 $\mathrm{AN}$ 陶瓷, 在 $175 \mathrm{kV} / \mathrm{cm}$ 的外加电场作用下, 其 $P_{\text {max }}$ 达到 $40 \mu \mathrm{C} / \mathrm{cm}^{2}, W_{\mathrm{rec}}$ 达到 $2.1 \mathrm{~J} / \mathrm{cm}^{3}$ 。在此基础上, Zhao 等 ${ }^{[95]}$ 通过引入适量的 $\mathrm{MnO}_{2}$ 来降低纯 $\mathrm{AN}$ 陶瓷的漏电流、增加致密性、提 高其温度稳定性并优化 $P-E$ 曲线, 最终 $W_{\text {rec }}$ 达到 $2.5 \mathrm{~J} / \mathrm{cm}^{3}$ 。最近, Zhao 等 ${ }^{[78]}$ 利用 $\mathrm{Ta}^{5+}$ 部分取代 $\mathrm{AN}$ 中 $\mathrm{B}$ 位的 $\mathrm{Nb}^{5+}$ 来提高材料的反铁电性, 结果表明引 入 $\mathrm{Ta}^{5+}$ 不仅能减小材料的 $P_{\mathrm{r}}$, 而且能提高反铁电铁电相变电场 $\left(E_{\mathrm{F}}\right)$ 和铁电一反铁电相变电场 $\left(E_{\mathrm{A}}\right)$, 同 时引入 $\mathrm{Ta}^{5+}$ 还可以减小晶粒尺寸, 提高陶瓷的致密 性, 进而提高其 $E_{\mathrm{b}}$, 最终, 厚度为 $0.2 \mathrm{~mm}$ 的样品在 $233 \mathrm{kV} / \mathrm{cm}$ 的外加电场作用下, $W_{\mathrm{rec}}$ 达到 $4.2 \mathrm{~J} / \mathrm{cm}^{3}$, 但其 $\eta$ 相对较低, 仅为 $69 \%$ 。总之, $\mathrm{AN}$ 基陶瓷作为一 种新型高 $W_{\mathrm{rec}}$ 介质材料, 已经显示出广阔的前景。

\subsection{2 $\left(\mathrm{Bi}_{0.5} \mathrm{Na}_{0.5}\right) \mathrm{TiO}_{3}(\mathrm{BNT})$ 基陶瓷}

$\mathrm{BNT}$ 是近 20 年来被广泛研究的无铅压电陶瓷 之一, 研究内容主要集中在如何提高其压电性能和 降低其去极化温度上 ${ }^{[96-98]}$ 。近几年, BNT 基陶瓷在 介电储能领域也受到了广泛关注, 但其铁电性较强 $\left(P_{\mathrm{r}}=38 \mu \mathrm{C} / \mathrm{cm}^{2}, E_{\mathrm{c}}=73 \mathrm{kV} / \mathrm{cm}\right)$, 同时纯 BNT 难以烧 成致密样品, 缺陷较多, 进而导致其 $E_{\mathrm{b}}$ 较低, 因此, 纯 BNT 难以用作储能介质。目前该材料体系的设计 思路为: 一方面通过 $A 、 B$ 位复合掺杂取代产生 PNRs, 这主要是由于 PNRs 的铁电畴相对较大, 在 放电完成后 $P_{\mathrm{r}}$ 较低, 从而能放出更多的能量, 如图 8

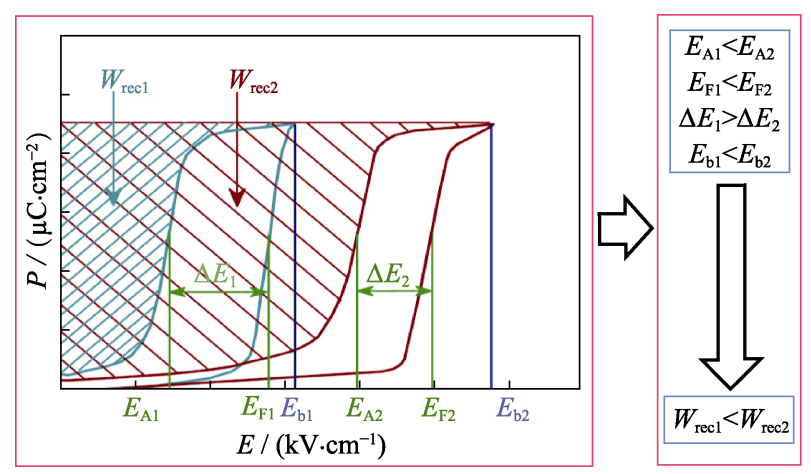

图 $7 \mathrm{AN}$ 基材料体系的组分设计思路图 ${ }^{[78]}$

Fig. 7 Schematic diagram of composition design of AN based material system ${ }^{[78]}$
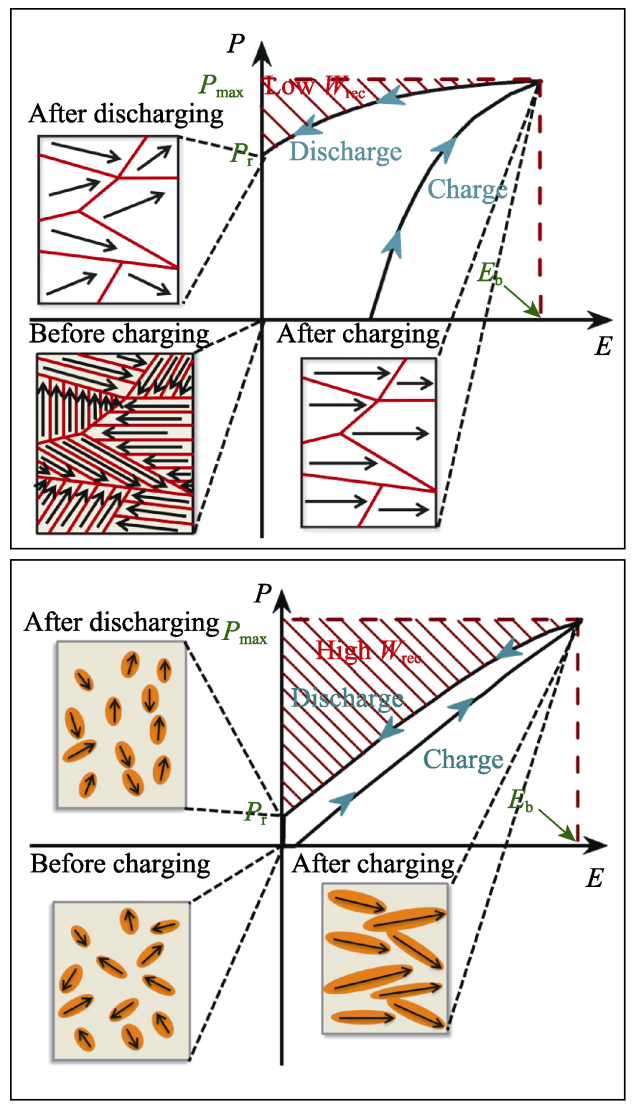

图 8 具有大铁电畴介质和具有极性纳米微区介质的充放电 过程对比图 ${ }^{[102-103]}$

Fig. 8 Comparison of charging and discharging process of dielectrics with large ferroelectric domain and with $\mathrm{PNRs}^{[102-103]}$

所示。另一方面通过添加烧结助剂或改进制备工艺 改善材料的显微结构, 提高其致密性, 从而获得高 $E_{\mathrm{b}}$, 最终提高材料的 $W_{\mathrm{rec}}$ 。

目前, BNT 基块体材料的研究主要集中在 $\left(\mathrm{Bi}_{0.5} \mathrm{Na}_{0.5}\right) \mathrm{TiO}_{3}-\mathrm{BaTiO}_{3}(\mathrm{BNT}-\mathrm{BT}) 、\left(\mathrm{Bi}_{0.5} \mathrm{Na}_{0.5}\right) \mathrm{TiO}_{3}-$ $\mathrm{SrTiO}_{3}(\mathrm{BNT}-\mathrm{ST}) 、\left(\mathrm{Bi}_{0.5} \mathrm{Na}_{0.5}\right) \mathrm{TiO}_{3}-\mathrm{KNbO}_{3}(\mathrm{BNT}-\mathrm{KN})$ 等体系。如: Gao 等 ${ }^{[99]}$ 用固相反应法制备了 0.89 $\left(\mathrm{Bi}_{0.5} \mathrm{Na}_{0.5}\right) \mathrm{TiO}_{3}-0.06 \mathrm{BaTiO}_{3}-0.05 \mathrm{~K}_{0.5} \mathrm{Na}_{0.5} \mathrm{NbO}_{3}(0.89 \mathrm{BNT}-$ $0.06 \mathrm{BT}-0.05 \mathrm{KNN}$ ) 陶瓷, 在 $56 \mathrm{kV} / \mathrm{cm}$ 的外加电场下, $W_{\mathrm{rec}}$ 达到了 $0.46 \mathrm{~J} / \mathrm{cm}^{3}$ 。为进一步提高其 $E_{\mathrm{b}}$, Ding 等 ${ }^{[100]}$ 通过改进制备工艺, 用两步烧结法制备了 0.89BNT$0.06 \mathrm{BT}-0.05 \mathrm{KNN}$ 陶瓷, 最终将 $0.89 \mathrm{BNT}-0.06 \mathrm{BT}-$ $0.05 \mathrm{KNN}$ 陶瓷的 $E_{\mathrm{b}}$ 由原来的 $56 \mathrm{kV} / \mathrm{cm}$ 提高到 $82 \mathrm{kV} / \mathrm{cm}, W_{\mathrm{rec}}$ 达到 $0.90 \mathrm{~J} / \mathrm{cm}^{3}, W_{\mathrm{rec}}$ 几乎提高一倍。 这是因为两步烧结法相对于单步烧结法可以制备出 致密度更高的细晶陶瓷, 从而有利于获得较高的 $E_{\mathrm{b}}$ 。这也说明 $E_{\mathrm{b}}$ 的提高对改进材料的 $W_{\mathrm{rec}}$ 效果十分 明显。Feng 等 ${ }^{[101]}$ 通过向 $(1-x)$ BNT $-x$ BT 陶瓷中添加 $\mathrm{BaO}-\mathrm{B}_{2} \mathrm{O}_{3}-\mathrm{SiO}_{2}$ 进行液相烧结, 提高陶瓷致密性来 提高材料的 $E_{\mathrm{b}}$, 最终 $W_{\mathrm{rec}}$ 达到 $0.68 \mathrm{~J} / \mathrm{cm}^{3}$ 。最近, Chen 等 ${ }^{[102]}$ 通过向 $0.92 \mathrm{BNT}-0.08 \mathrm{BT}$ 陶瓷中引入 
$\mathrm{Bi}\left(\mathrm{Mg}_{1 / 2} \mathrm{Ti}_{1 / 2}\right) \mathrm{O}_{3}(\mathrm{BMT})$, 发现 BMT 在降低材料铁电 性和 $\tan \delta$ 的同时还能提高材料的温度稳定性, 在厚 度为 $0.5 \mathrm{~mm}$ 的样品中, 其 $E_{\mathrm{b}}$ 达到 $135 \mathrm{kV} / \mathrm{cm}, W_{\mathrm{rec}}$ 达到 $2 \mathrm{~J} / \mathrm{cm}^{3}$, 并在 $25 \sim 180^{\circ} \mathrm{C}$ 范围内具有很好的储能 性能。此外, Cao 等 ${ }^{[103]}$ 通过向 BNT 陶瓷引入 $\mathrm{ST}$, 将 BNT 陶瓷的退极化温度降低到室温, 在退极化温度 附近 BNT 陶瓷存在铁电一反铁电相变, 因而具有很 大的 $\Delta P$, 进而获得较高的 $W_{\mathrm{rec}}\left(0.65 \mathrm{~J} / \mathrm{cm}^{3}\right)$, 并且该 材料可以在 $30 \sim 120^{\circ} \mathrm{C}$ 范围内保持很好的储能特性。 为进一步通过增大 $\Delta P$ 来获得高的 $W_{\mathrm{rec}}$, Cao 等 ${ }^{[103]}$ 选择具有高 $P_{\max }$ 的 0.94BNT-0.06BT 陶瓷作为基体材 料, 向其中引入 $\mathrm{ST}$, 最终制备了高 $W_{\mathrm{rec}}$ 的 $0.7(0.94 \mathrm{BNT}-$ $0.06 \mathrm{BT}$ )- $0.3 \mathrm{ST}$ 陶瓷, 其 $\Delta P$ 高达 $30.8 \mu \mathrm{C} / \mathrm{cm}^{2}, W_{\mathrm{rec}}$ 达 到 $0.98 \mathrm{~J} / \mathrm{cm}^{3}$, 此时 $\eta$ 为 $82 \%$ 。表 4 总结了 $\mathrm{AN}$ 和 $\mathrm{BNT}$ 基无铅反铁电陶瓷材料的储能性能。

\section{3 挑战}

探寻具有高 $W_{\mathrm{rec}}$ 和 $\eta$ 的储能介质材料是当今功 能陶瓷研究的热点之一, 通过近几年的努力, 无铅 储能陶瓷介质材料的研究取得了显著的进步。无铅 块体陶瓷的 $W_{\mathrm{rec}}$ 从 $1 \mathrm{~J} / \mathrm{cm}^{3}$ 提高到 $2 \mathrm{~J} / \mathrm{cm}^{3}$, 目前已经 达到 $4 \mathrm{~J} / \mathrm{cm}^{3}$ 。为了找出目前无铅储能陶瓷研究中存 在的问题, 进一步提高无铅陶瓷介质的储能特性探 索新的研究思路, 本文归纳和分析了一些有代表性 无铅块体陶瓷的储能性能。图 9 是无铅块体陶瓷的 $W_{\mathrm{rec}}$ 与 $E_{\mathrm{b}}$ 和 $W_{\mathrm{rec}}$ 与 $\eta$ 的关系图, 通过图中对比, 尝试
总结了无铅块体材料在介电储能领域所面临的一些 挑战及下一步发展方向:

（1）对于 $\mathrm{KNN}$ 基陶瓷而言，相对于其他材料体 系, 它具有较高的 $W_{\mathrm{rec}}\left(\sim 4 \mathrm{~J} / \mathrm{cm}^{3}\right)$, 主要得益于其高的 $E_{\mathrm{b}}(\sim 300 \mathrm{kV} / \mathrm{cm})$, 然而其 $\eta$ 还相对较低 $(<60 \%)$, 温度 适用范围还较窄，未来应该在降低其 $W_{\mathrm{rec}}$ 的基础上 适当提高 $\eta$ 并拓宽其温度适用范围。此外, $\mathrm{KNN}$ 基陶 瓷高的 $E_{\mathrm{b}}$ 主要得益于其亚微米级晶粒, 但是关于亚 微米级晶粒的生长机理尚不清楚，还有待研究; 同 时介电固态电容器要求介质材料不仅具有高的 $W_{\text {rec }}$ 、 高的 $\eta$ 、宽的温度适用范围而且应该具有较好的抗疲 劳特性和力学性能。因此 KNN 基陶瓷的抗疲劳特性 和力学性能也有待研究。而在 KNN 基陶瓷制备方面, 其烧结温区窄，钾钠元素的挥发，工艺重复性差等 问题也急需解决 ${ }^{[12]}$ 。在应用方面, KNN 基陶瓷不仅 具有高 $W_{\mathrm{rec}}$ 而且具有很好的透光性，需要为其新性能 (高 $W_{\mathrm{rec}}$ 和高透光性) 寻找新的应用领域。

（2） $\mathrm{BT}$ 基陶瓷，虽然具有中等的 $E_{\mathrm{b}}$ 但其较低的 $\Delta P$ 导致其 $W_{\mathrm{rec}}$ 相对较低, 而且其烧结温度偏高 $\left(>1200^{\circ} \mathrm{C}\right)$, 不易于批量生产。然而 $\mathrm{BT}$ 基陶瓷在 $\eta$ 和 温度稳定性方面具有明显的优势。未来应该在保持 $E_{\mathrm{b}}$ 的基础上进一步提高 $\Delta P$ 来提高其 $W_{\mathrm{rec}}$, 同时尽可 能降低其烧结温度。大的 $\Delta P$ 一方面可以利用 $\mathrm{A}$ 或 $\mathrm{B}$ 位掺杂增强其弛豫特性来获得，另一方面可以通过 与其他具有高 $\Delta P$ 的介质材料复合。

(3) 对于 $\mathrm{BNT}$ 基、 $\mathrm{BF}$ 基、 $\mathrm{AN}$ 基陶瓷而言, 其 共同优势是具有大 $\Delta P$, 但 $E_{\mathrm{b}}$ 相对较低, 未来应该在

表 4 AN 和 BNT 基无铅反铁电陶瓷的储能性能

Table 4 Energy storage properties of AN-based and BNT-based lead-free anti-ferroelectric

\begin{tabular}{|c|c|c|c|c|c|c|}
\hline Materials & Compositions & $E_{\mathrm{b}} /\left(\mathrm{kV} \cdot \mathrm{cm}^{-1}\right)$ & $\Delta P /\left(\mu \mathrm{C} \cdot \mathrm{cm}^{-2}\right)$ & $W_{\mathrm{rec}} /\left(\mathrm{J} \cdot \mathrm{cm}^{-3}\right)$ & $\eta / \%$ & Ref. \\
\hline \multirow{12}{*}{$\mathrm{BNT}$} & $0.89 \mathrm{BNT}-0.06 \mathrm{BT}-0.05 \mathrm{KNN}$ & 99 & 27.2 & 0.90 & - & {$[100]$} \\
\hline & $0.95 \mathrm{BNT}-0.05 \mathrm{BT}+3 \% \mathrm{BaO}-\mathrm{B}_{2} \mathrm{O}_{3}-\mathrm{SiO}_{2}$ & 94.6 & 31 & 0.68 & 71 & [101] \\
\hline & $0.9[0.92 \mathrm{BNT}-0.08 \mathrm{BT}]-0.1 \mathrm{BMT}$ & 135 & $\sim 40$ & 2.00 & 88 & {$[102]$} \\
\hline & $0.7 \mathrm{BNT}-0.3 \mathrm{ST}+0.05 \mathrm{MnO}_{2}$ & 95 & 36.92 & 0.96 & 74.6 & {$[103]$} \\
\hline & $0.9[0.92 \mathrm{BNT}-0.08 \mathrm{BT}]-0.1 \mathrm{NaNbO}_{3}$ & 70 & 25 & 0.71 & 66 & [104] \\
\hline & $0.7[0.94 \mathrm{BNT}-0.06 \mathrm{BT}]-0.3 \mathrm{ST}$ & 90 & 30.8 & 0.98 & 82 & {$[105]$} \\
\hline & 0.6BNT-0.2NN-0.2 Ba $\left(\mathrm{Zr}_{0.2} \mathrm{Ti}_{0.8}\right) \mathrm{O}_{3}$ & 175 & 26.61 & 1.69 & 78.4 & {$[106]$} \\
\hline & $0.9 \mathrm{BNT}-0.1 \mathrm{KN}$ & 104 & 28 & 1.17 & 83 & [107] \\
\hline & $0.84 \mathrm{BNT}-0.16 \mathrm{KNN}$ & 100 & - & 1.20 & 71 & [108] \\
\hline & {$\left[\left(\mathrm{Bi}_{1 / 2} \mathrm{Na}_{1 / 2}\right)_{0.94} \mathrm{Ba}_{0.06}\right] \mathrm{La}_{0.98} \mathrm{Zr}_{0.02} \mathrm{TiO}_{3}$} & 83.4 & 33 & 0.58 & - & {$[109]$} \\
\hline & 0.62BNT-0.06BT-0.32( $\left(\mathrm{Sr}_{0.7} \mathrm{Bi}_{0.3}\right) \mathrm{TiO}_{3}$ & 60 & $\sim 19$ & 0.5 & $>90$ & [110] \\
\hline & $0.95(0.8 \mathrm{BNT}-0.2 \mathrm{ST})-0.05 \mathrm{NaNbO}_{3}$ & 70 & 27.53 & 0.74 & 55 & [111] \\
\hline \multirow{3}{*}{ AN } & AN & 175 & - & 2.1 & - & [94] \\
\hline & $\mathrm{AN}+0.1 \mathrm{wt} \% \mathrm{MnO}_{2}$ & 150 & $\sim 37$ & 2.5 & 56 & {$[95]$} \\
\hline & $\mathrm{Ag}\left(\mathrm{Nb}_{0.85} \mathrm{Ta}_{0.15}\right) \mathrm{O}_{3}$ & 233 & 36.1 & 4.2 & 69 & {$[78]$} \\
\hline
\end{tabular}



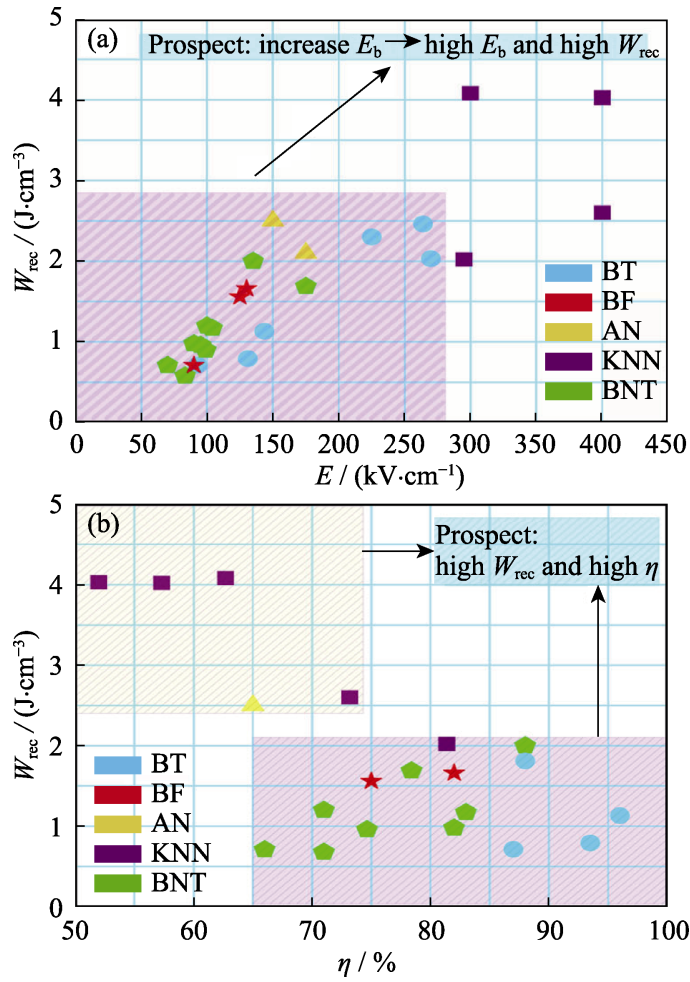

图 9 典型无铅块体陶瓷的储能性 $[23,48,51-53,60-62,84-86,90-91,94-95,101-108]$

Fig. 9 Typical lead-free bulk ceramics ${ }^{[23,48,51-53,60-62,84-86,90-91,94-95,101-108]}$ (a) $W_{\text {rec }}$ and $E_{\mathrm{b}}$, (b) $W_{\text {rec }}$ and $\eta$

保持大 $\Delta P$ 的基础上, 进一步提高它们的 $E_{\mathrm{b}}$ 来提高

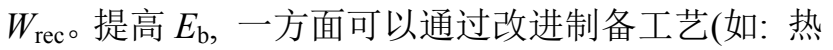
压烧结法、两步烧结法、多次球磨和预烧法), 另一 方面可以通过掺杂具有高 $E_{\mathrm{b}}$ 的氧化物(如: 玻璃粉, $\mathrm{MgO}, \mathrm{Al}_{2} \mathrm{O}_{3}$ )制备复合材料来实现。此外, $\mathrm{BF}$ 基陶瓷 还存在损耗大和钻钛矿结构稳定性较差的问题; $\mathrm{AN}$ 基陶瓷还存在 $\eta$ 偏低、制备困难和成本高的问题。

\section{参考文献:}

[1] HAO X H. A review on the dielectric materials for high energystorage application. J. Adv. Dielect., 2013(1): 1330001- 1330014.

[2] YAO L M, PAN Z B, LIU S H, et al. Significantly enhanced energy density in nanocomposite capacitors combining the $\mathrm{TiO}_{2}$ nanorod array with poly(vinylidene fluoride). ACS Appl. Mater. \& Interfaces, 2016, 8(39): 26343-26351.

[3] HOU C M, HUANG W C, ZHAO W B, et al. Ultrahigh energy density in $\mathrm{SrTiO}_{3}$ film capacitors. ACS Appl. Mater. \& Interfaces, 2017, 9(24): 20484-20490.

[4] CHANHAN A, PATEL S, VAISH R, et al. Anti-ferroelectric ceramics for high energy density capacitors. Materials, 2015, 8(12): 8009-8031.

[5] YAO Z H, SONG Z, HAO H, et al. Homogeneous/ inhomogeneous-structured dielectrics and their energy-storage performances. Adv. Mater., 2017, 29(20): 1601727-1601741.

[6] WEI W, YAN H, WANG T, et al. Reverse boundary layer capacitor model in glass/ceramic composites for energy storage applications. J. Appl. Phys., 2013, 113(2): 024103-024107.

[7] TOPRAK A, TIGLI O. Piezoelectric energy harvesting: stateof-the-art and challenges. Appl. Phys. Rev., 2014, 1(3): $031104-$
031117.

[8] LI Y X, XIE J L, CHU Z M, et al. Dielectric and energy storage properties of ceramic/PVDF composites with titanate coupling agents. Ferroelectrics, 2013, 452(1): 101-106.

[9] YI D, YUAN J C, LIU H Y, et al. Influence of $\mathrm{Al}_{2} \mathrm{O}_{3}$ additive on the dielectric behavior and energy density of $\mathrm{Ba}_{0.5} \mathrm{Sr}_{0.5} \mathrm{TiO}_{3}$ ceramics. J. Electroceram., 2012, 29(2): 95-98.

[10] HUANG Y H, WU Y J, QIU W J, et al. Enhanced energy storage density of $\mathrm{Ba}_{0.4} \mathrm{Sr}_{0.6} \mathrm{TiO}_{3}-\mathrm{MgO}$ composite prepared by spark plasma sintering. J. Eur. Ceram. Soc., 2015, 35(5): 1469-1476.

[11] LI YONG-XIANG. Some hot topics in electroceramics research. Journal of Inorganic Materials, 2014, 29(1): 1-5.

[12] LI J J, PAISAN K, HAN K, et al. New route toward high-energy-density nanocomposites based on chain-end functionalized ferroelectric polymers. Chem. Mater, 2010, 22(18): 5350- 5357.

[13] PARIZI S S, MELLINGER A, CARUNTU G, et al. Ferroelectric barium titanate nanocubes as cordially, apacitive building best wishes, locks for energy storage applications. ACS Appl. Mater. \& Interfaces, 2014, 6(20): 17506-17517.

[14] WU T, PU Y P, ZONG T T, et al. Microstructures and dielectric properties of $\mathrm{Ba}_{0.4} \mathrm{Sr}_{0.6} \mathrm{TiO}_{3}$ ceramics with $\mathrm{BaO}-\mathrm{TiO}_{2}-\mathrm{SiO}_{2}$ glassceramics addition. J. Alloy. Compd., 2014, 584(25): 461-465.

[15] NICHOLAS J S, BADRI R, MICHAEL T L, et al. Alkali-free glass as a high energy density dielectric material. Mater. Lett., 2009, 63(15): 1245-1248.

[16] ACOSTA M, ZANG J D, JO W, et al. High-temperature dielectrics in $\mathrm{CaZrO}_{3}$-modified $\mathrm{Bi}_{1 / 2} \mathrm{Na}_{1 / 2} \mathrm{TiO}_{3}$-based lead-free ceramics. J. Eur. Ceram. Soc., 2012, 32(16): 4327-4334.

[17] RAENGTHON N, SEBASTIAN T, RAENGTHON N N, et al. $\mathrm{BaTiO}_{3}-\mathrm{Bi}\left(\mathrm{Zn}_{1 / 2} \mathrm{Ti}_{1 / 2}\right) \mathrm{O}_{3}-\mathrm{BiScO}_{3}$ ceramics for high-temperature capacitor applications. J. Am. Ceram. Soc., 2012, 95(11): 3554 3561.

[18] MUHAMMAD R, IQBAL Y, REANEY I M, et al. $\mathrm{BaTiO}_{3}$ $\mathrm{Bi}\left(\mathrm{Mg}_{2 / 3} \mathrm{Nb}_{1 / 3}\right) \mathrm{O}_{3}$ ceramics for high-temperature capacitor applications. J. Am. Ceram. Soc., 2016, 99(6): 2089-2095.

[19] WANG X L, ZHANG L, HAO X H, et al. High energy-storage performance of $0.9 \mathrm{~Pb}\left(\mathrm{Mg}_{1 / 3} \mathrm{Nb}_{2 / 3}\right) \mathrm{O}_{3}-0.1 \mathrm{PbTiO}_{3}$ relaxor ferroelectric thin films prepared by RF magnetron sputtering. Mater. Res. Bull., 2015, 65: 73-79.

[20] PENG B L, ZHANG Q, LI X, et al. Giant electric energy density in epitaxial lead-free thin films with coexistence of ferroelectrics and antiferroelectrics. Adv. Electron. Mater, 2015, 1(5): 1500052-1500058.

[21] WANG J H, SUN N N, LI Y, et al. Effects of mn doping on dielectric properties and energy-storage performance of $\mathrm{Na}_{0.5} \mathrm{Bi}_{0.5} \mathrm{TiO}_{3}$ thick films. Ceram. Int., 2017, 43(10): 7804-7809.

[22] CHAO S, DOGAN F. Processing and dielectric properties of $\mathrm{TiO}_{2}$ thick films for high-energy density capacitor applications. Int. J. Appl. Ceram. Tec., 2011, 8(6): 1363-1373.

[23] HU Q Y, JIN L, WANG T, et al. Dielectric and temperature stable energy storage properties of $0.88 \mathrm{BaTiO}_{3}-0.12 \mathrm{Bi}\left(\mathrm{Mg}_{1 / 2} \mathrm{Ti}_{1 / 2}\right) \mathrm{O}_{3}$ bulk ceramics. J. Alloy. Compd., 2015, 640: 416-420.

[24] KWON D K, LEE H M. Temperature stable high energy density capacitors using complex perovskite thin films. IEEE. T. Ultrason. Ferr., 2012, 59(9): 1894-1899.

[25] DAMJANOVIC D, KLEIN D, LI J, et al. What can be expected from lead-free piezoelectric materials. Funct. Mater. Lett., 2010, 3(1): 5-13.

[26] TAKENAKA T, NAGATA H. Current status and prospects of lead-free piezoelectric ceramics. J. Eur. Ceram. Soc., 2005, 25(12): 2693-2700. 
[27] WANG YA-JUN, WU XIAO-JUAN, ZENG QING-XUAN. Review on barium titanate based composites with high energy storage density. Science\&Technology Review, 2012, 30(10): 65-71.

[28] GU YI-TAO, LIU HONG-BO, MA HAI-HUA, et al. Research progress of dielectric materials for energy storage. Insulating Materials, 2015, 48(11): 1-13.

[29] BURN I, SMYTH D M. Energy storage in ceramic dielectrics. $J$. Mater. Sci., 1972, 7(3): 339-343.

[30] LOVE G R. Energy storage in ceramic dielectric. J. Am. Ceram. Soc., 1990, 73(2): 323-328.

[31] FLETCHER N H, HILTON A D, RICKETTS B W. Optimization of energy storage density in ceramic capacitors. J. Phys. D: Appl. Phys., 1996, 29: 253-258.

[32] ZHANG L, HAO X H, ZHANG L W. Enhanced energy-storage performances of $\mathrm{Bi}_{2} \mathrm{O}_{3}-\mathrm{Li}_{2} \mathrm{O}$ added $(1-x)\left(\mathrm{Na}_{1 / 2} \mathrm{Bi}_{1 / 2}\right) \mathrm{TiO}_{3}-x \mathrm{BaTiO}_{3}$ thick films. Ceram. Int., 2014, 40(6): 8847-8851.

[33] XIU S M, XIAO S, XUE S X, et al. Effect of different Al/Si ratios on the structure and energy storage properties of strontium barium niobate-based glass-ceramics. J. Electron. Mater, 2016, 45(2): 1017-1022.

[34] ZHAO X J, PENG G R, ZHAN Z J, et al. Structure change and energy storage property of poly(vinylidene fluoride hexafluoropropylene)/poly(methyl methacrylate) blends. Polym. Sci. Ser. A., 2015, 57(4): 452-459.

[35] WEI M, ZHANG J H, LIU J F, et al. Effect of multiple times pre-sintering on the dielectric properties of $\mathrm{TiO}_{2} /$ glass composite. J. Mater. Sci-Mater. El., 2017, 28(1): 526-531.

[36] SHAY D P, PODRAZA N J, DONNELLY N J, et al. High energy density, high temperature capacitors utilizing mn-doped $0.8 \mathrm{CaTiO}_{3}$ 0.2 $\mathrm{CaHfO}_{3}$ Ceramics. J. Am. Ceram. Soc., 2012, 95(4): 1348- 1355.

[37] JIANG S, ZHANG L, ZHANG G, et al. Effect of Zr: Sn ratio in the lead lanthanum zirconate stannate titanate anti-ferroelectric ceramics on energy storage properties. Ceram. Int., 2013, 39(5): 5571- 5575 .

[38] DANG Z M, YUAN J K, YAO S H, et al. Flexible nanodielectric materials with high permittivity for power energy storage. $A d v$. Mater, 2013, 25(44): 6334-6365.

[39] QING Y C, WEN Q L, LUO F, et al. Graphene nanosheets/BaTiO ceramics as highly efficient electromagnetic interference shielding materials in the x-band. J. Mater. Chem. C, 2016, 4(2): 371-375.

[40] TAKASHI T, TAKUYA H, HIROAKI T, et al. Fabrication of lead-free semiconducting ceramics using $\mathrm{BaTiO}_{3}-\left(\mathrm{Bi}_{1 / 2} \mathrm{Na}_{1 / 2}\right) \mathrm{TiO}_{3}$ system by adding CaO. J. Ceram. Soc. Jpn., 2011, 119(1395): $828-831$

[41] WANG X R, ZHANG Y, SONG X Z, et al. Glass additive in barium titanate ceramics and its influence on electrical breakdown strength in relation with energy storage properties. J. Eur. Ceram. Soc., 2012, 32(3): 559-567.

[42] YOUNG A, HILMAS G, ZHANG S C, et al. Effect of liquidphase sintering on the breakdown strength of barium titanate. $J$. Am. Ceram. Soc., 2007, 90(5): 1504-1510.

[43] SU X F, RIGGS B C, TOMOZAWA M, et al. Preparation of $\mathrm{BaTiO}_{3} /$ low melting glass core-shell nanoparticles for energy storage capacitor applications. J. Mater. Chem. A, 2014, 2(42): 18087-18096.

[44] EJAZ M, PULI VS, ELUPULA R, et al. Core-shell structured poly(glycidyl methacrylate) $/ \mathrm{BaTiO}_{3}$ nanocomposites prepared by surface-initiated atom transfer radical polymerization: a novel material for high energy density dielectric storage. J. Polym. Sci. Pol. Chem., 2015, 53(6): 719-728.

[45] KWON D K, LEE H M. Temperature stable high energy density capacitors using complex perovskite thin films. IEEE. T. Ultrason. Ferr., 2012, 59(9): 1894-1899.

[46] HUANG X Y, JIANG P K. Core-shell structured high-k polymer nanocomposites for energy storage and dielectric applications. Adv. Mater., 2015, 27(3): 546-554.

[47] XU W H, DING Y C, JIANG S H, et al. Polyimide $/ \mathrm{BaTiO}_{3} /$ MWCNTs three-phase nanocomposites fabricated by electrospinning with enhanced dielectric properties. Mater. Lett., 2014, 135: 158-161.

[48] OGIHARA H, RANDALL C A, SUSAN T M. High-energy density capacitors utilizing $0.7 \mathrm{BaTiO}_{3}-0.3 \mathrm{BiScO}_{3}$ ceramics. $J . \mathrm{Am}$. Ceram. Soc., 2009, 92(8): 1719-1724.

[49] NEUSEL C, JELITTO H, SCHNEIDER GA. Electrical conduction mechanism in bulk ceramic insulators at high voltages until dielectric breakdown. J. Appl. Phys., 2015, 117(15): 154902 154910.

[50] LIM J B, ZZHANG S J, KIM N, et al. High-temperature dielectrics in the $\mathrm{BiScO}_{3}-\mathrm{BaTiO}_{3}-\left(\mathrm{K}_{1 / 2} \mathrm{Bi}_{1 / 2}\right) \mathrm{TiO}_{3}$ ternary system. $J$. Am. Ceram. Soc., 2009, 92(3): 679-682.

[51] WANG T, JIN L, LI C C, et al. Relaxor ferroelectric $\mathrm{BaTiO}_{3}$ $\mathrm{Bi}\left(\mathrm{Mg}_{2 / 3} \mathrm{Nb}_{1 / 3}\right) \mathrm{O}_{3}$ ceramics for energy storage application. J. Am. Ceram. Soc., 2015, 98(2): 559-566.

[52] SHEN Z B, WANG X H, LUO B C, et al. $\mathrm{BaTiO}_{3}-\mathrm{BiYbO}_{3}$ perovskite materials for energy storage applications. J. Mater. Chem. A, 2015, 3(35): 18146-18153.

[53] WU L W, WANG X H, LI L T, et al. Lead-free $\mathrm{BaTiO}_{3}$ $\mathrm{Bi}\left(\mathrm{Zn}_{2 / 3} \mathrm{Nb}_{1 / 3}\right) \mathrm{O}_{3}$ weakly coupled relaxor ferroelectric materials for energy storage. RSC Adv., 2016, 6(17): 14273-14282.

[54] LI W B, ZHOU D, PANG L X. Novel barium titanate based capacitors with high energy density and fast discharge performance. J. Mater. Chem. A, 2017, 5(37): 19607-19612.

[55] YUAN Q B, YAO F Z, WANG Y F. Relaxor-ferroelectric $0.9 \mathrm{BaTiO}_{3}-0.1 \mathrm{Bi}\left(\mathrm{Zn}_{1 / 2} \mathrm{Zr}_{1 / 2}\right) \mathrm{O}_{3}$ ceramics capacitors with high energy density and temperature stable energy storage properties. $J$. Mater. Chem. C, 2017, 5(37): 9552-9558.

[56] XU R, XU Z, FENG Y J, et al. Temperature dependence of energy storage in $\mathrm{Pb}_{0.90} \mathrm{La}_{0.04} \mathrm{Ba}_{0.04}\left[\left(\mathrm{Zr}_{0.7} \mathrm{Sn}_{0.3}\right)_{0.88} \mathrm{Ti}_{0.12}\right] \mathrm{O}_{3}$ antiferroelectric ceramics. J. Am. Ceram. Soc., 2016, 99(9): 2984-2988.

[57] MOREAU J M, MICHEL C, GERSON R, et al. Ferroelectric $\mathrm{BiFeO}_{3} \mathrm{X}$-ray and neutron diffraction study. J. Phys. Chem. Solids., 1971, 32(6): 1315-1335.

[58] TEAGUE J R, GERSON R, JAMES W J. Dielectic hysteresis in single crystal $\mathrm{BiFeO}_{3}$. Solid State Commun., 1970, 8(13): 1073- 1074.

[59] KHANSUR N H, ROJAC T, DAMJANOVIC D, et al. Electricfield-induced domain switching and domain texture relaxations in bulk bismuth ferrite. J. Am. Ceram. Soc., 2015, 98(12): 3884 3890.

[60] WANG T, JIN L, TIAN Y, et al. Microstructure and ferroelectric properties of $\mathrm{Nb}_{2} \mathrm{O}_{5}$-modified $\mathrm{BiFeO}_{3}-\mathrm{BaTiO}_{3}$ lead-free ceramics for energy storage. Mater. Lett., 2014, 137: 79-81.

[61] ZHENG D, ZUO R D. Enhanced energy storage properties in $\mathrm{La}\left(\mathrm{Mg}_{1 / 2} \mathrm{Ti}_{1 / 2}\right) \mathrm{O}_{3}$-modified $\mathrm{BiFeO}_{3}-\mathrm{BaTiO}_{3}$ lead-free relaxor ferroelectric ceramics within a wide temperature range. J. Eur. Ceram. Soc., 2017, 37(1): 413-418.

[62] ZHENG D G, ZUO R Z, ZHANG D S, et al. Novel $\mathrm{BiFeO}_{3-}$ $\mathrm{BaTiO}_{3}-\mathrm{Ba}\left(\mathrm{Mg}_{1 / 3} \mathrm{Nb}_{2 / 3}\right) \mathrm{O}_{3}$ lead-free relaxor ferroelectric ceramics for energy-storage capacitors. J. Am. Ceram. Soc., 2015, 98(9): 2692-2695.

[63] CORREIA T M, MILLEN M M, ROKOSZ M K. A lead-free and high-energy density ceramic for energy storage applications. $J$. Am. Ceram. Soc., 2013, 96(9): 2699-2702.

[64] CORREIA T, STEWART M, ELLMORE A, et al. Lead-free ce- 
ramics with high energy density and reduced losses for high temperature applications. Adv. Eng. Mater, 2017, 19(6): 1700019-1-5.

[65] PAN H, ZEENG Y, SHEN Y, et al. $\mathrm{BiFeO}_{3}-\mathrm{SrTiO}_{3}$ thin film as a new lead-free relaxor ferroelectric capacitor with ultrahigh energy storage performance. J. Mater. Chem. A, 2017, 5(12): 5920-5926.

[66] CHEN X L, HE F, CHEN J, et al. An approach to further improve piezoelectric and ferroelectric properties of $\left(\mathrm{K}_{0.5} \mathrm{Na}_{0.5}\right) \mathrm{NbO}_{3}$ ceramic. J. Mater. Sci-Mater. El., 2014, 25(6): 2634-2637.

[67] DU H L, LIU D J, TANG F S, et al. Microstructure, piezoelectric, and ferroelectric properties of $\mathrm{Bi}_{2} \mathrm{O}_{3}$-added $\left(\mathrm{K}_{0.5} \mathrm{Na}_{0.5}\right) \mathrm{NbO}_{3}$ leadfree ceramics. J. Am. Ceram. Soc., 2007, 90(9): 2824-2829.

[68] LI F L, KWOK K W. $\mathrm{K}_{0.5} \mathrm{Na}_{0.5} \mathrm{NbO}_{3}$-based lead-free transparent electro-optic ceramics prepared by pressureless sintering. J. Am. Ceram. Soc., 2013, 96(11): 3557-3562.

[69] WU X, LU S B, KWOK K W, et al. Photoluminescence, electrooptic response and piezoelectric properties in pressureless- sintered Er-doped KNN-based transparent ceramics. J. Alloy. Compd., 2017, 695: 3573-3578.

[70] LI J T, BAI Y, QIN S Q. Direct and indirect characterization of electrocaloric effect in $(\mathrm{Na}, \mathrm{K}) \mathrm{NbO}_{3}$ based lead-free ceramics. Appl. Phys. Lett., 2016, 109(16): 162902-162904.

[71] WANG X J, WU J G, BRAHIM D. Enhanced electrocaloric effect near polymorphic phase boundary in lead-free potassium sodium niobate ceramics. Appl. Phys. Lett., 2017, 110(6): 063904063908 .

[72] ZHANG G F, LIU H X, YAO Z H, et al. Effects of Ca doping on the energy storage properties of $(\mathrm{Sr}, \mathrm{Ca}) \mathrm{TiO}_{3}$ paraelectric ceramics. J. Mater. Sci: Mater. Electron., 2015, 26(5): 2726-2732.

[73] NEUSEL C, SCHNEIDER G. Size-dependence of the dielectric breakdown strength from nano-to millimeter scale. J. Mech. Phys. Solids., 2014, 63: 201-213.

[74] TUNKASIRI T, RUJIJANAGUL G. Dielectric strength of fine grained barium titanate ceramics. J. Mater. Sci. Lett., 1996, 15(20): 1767-1769.

[75] SONG Z, LIU H X, ZHANG S J, et al. Effect of grain size on the energy storage properties of $\left(\mathrm{Ba}_{0.4} \mathrm{Sr}_{0.6}\right) \mathrm{TiO}_{3}$ paraelectric ceramics. J. Eur. Ceram. Soc., 2014, 34(5): 1209-1217.

[76] YE Y, ZHANG S C, DOGAN F, et al. Influence of nanocrystalline grain size on the breakdown strength of ceramic dielectrics. Proc-IEEE Int. Pulsed Power Conf., 2003, 1: 719-722.

[77] LEE H Y, CHO K H, NAM H D. Grain size and temperature dependence of electrical breakdown in $\mathrm{BaTiO}_{3}$ ceramic. Ferroelectrics, 2006, 334(1): 165-169.

[78] ZHAO L, LIU Q, GAO J, et al. Lead-free antiferroelectric silver niobate tantalate with high energy storage performance. $A d v$. Mater., 2017, 29(31): 1701824-1701831.

[79] HUANG Y H, WU Y J, QIU W J, et al. Enhanced energy storage density of $\mathrm{Ba}_{0.4} \mathrm{Sr}_{0.6} \mathrm{TiO}_{3}-\mathrm{MgO}$ composite prepared by spark plasma sintering. J. Eur. Ceram. Soc., 2015, 35(5): 1469-1476.

[80] ZHANG G Z, ZHU D Y, ZHANG X S, et al. High-energy storage performance of $\left(\mathrm{Pb}_{0.87} \mathrm{Ba}_{0.1} \mathrm{La}_{0.02}\right)\left(\mathrm{Zr}_{0.68} \mathrm{Sn}_{0.24} \mathrm{Ti}_{0.08}\right) \mathrm{O}_{3}$ antiferroelectric ceramics fabricated by the hot-press sintering method. $J$. Am. Ceram. Soc., 2015, 98(4): 1175-1181.

[81] KOSEC M, BOBNAR V, HROVAT M, et al. New lead-free relaxors based on the $\mathrm{K}_{0.5} \mathrm{Na}_{0.5} \mathrm{NbO}_{3}-\mathrm{SrTiO}_{3}$ solid solution. J. Mater. Res., 2004, 19(6): 1849-1854.

[82] LIU Z Y, FANH Q, ZHAO Y W, et al. Optical and tunable dielectric properties of $\mathrm{K}_{0.5} \mathrm{Na}_{0.5} \mathrm{NbO}_{3}-\mathrm{SrTiO}_{3}$ ceramics. J. Am. Ceram. Soc., 2016, 99(1): 146-151.

[83] WANG BIN-KE, TIAN XIAO-XIA, XU ZHUO, et al. Preparation and performances of KNN-based lead-free transparent ce- ramics. Acta Phys. Sin., 2012, 61(19): 197703-197707.

[84] QU B Y, DU H L, YANG Z T. Lead-free relaxor ferroelectric ceramics with high optical transparency and energy storage ability. $J$. Mater. Chem. C, 2016, 4(9): 1795-1803.

[85] YANG Z T, DU H L, QU S B, et al. Significantly enhanced recoverable energy storage density in potassium-sodium niobatebased lead free ceramics. J. Mater. Chem. A, 2016, 4(36): 13778-13785.

[86] SHAO T Q, DU H L, MA H, et al. Potassium-sodium niobate based lead-free ceramics: novel electrical energy storage materials. J. Mater. Chem. A, 2017, 5(2): 554-563.

[87] DONG G X, MA S W, DU J, et al. Dielectric properties and energy storage density in $\mathrm{ZnO}$-doped $\mathrm{Ba}_{0.3} \mathrm{Sr}_{0.7} \mathrm{TiO}_{3}$ ceramics. Ceram. Int., 2009, 35(5): 2069-2075.

[88] GERMAN R M, SURI P, PARK S J. Review: liquid phase sintering. J. Mater. Sci., 2009, 44(1): 1-39.

[89] ZHANGQ M, WANG L, LUO J, et al. Improved energy storage density in barium strontium titanate by addition of BaO- $-\mathrm{SiO}_{2}-\mathrm{B}_{2} \mathrm{O}_{3}$ glass. J. Am. Ceram. Soc., 2009, 92(8): 1871-1873.

[90] QU B Y, DU H L, YANG Z T, et al. Enhanced dielectric breakdown strength and energy storage density in lead-free relaxor ferroelectric ceramics prepared using transition liquid phase sintering. RSC Adv., 2016, 6(41): 34381-34389.

[91] QU B Y, DU H L, YANG Z T, et al. Large recoverable energy storage density and low sintering temperature in potassiumsodium niobate based ceramics for multilayer pulsed power capacitors. J. Am. Ceram. Soc., 2017, 100(4): 1517-1526.

[92] SHIGEMI A, WADA T. Crystallographic phase stabilities and electronic structures in $\mathrm{AgNbO}_{3}$ by first-principles calculation. Mol. Simulat., 2008, 34(10-15): 1105-1114.

[93] VERWERFT M, DYCK DV, BRABERS V M, et al. Electron microscopic study of the phase transformations in $\mathrm{AgNbO}_{3}$. Phys. Status. Solidi. A, 1989, 112(2): 451-466.

[94] TIAN Y, JIN L, ZHANG H F, et al. High energy density in silver niobate ceramics. J. Mater. Chem. A, 2016, 4(44): 17279-17288.

[95] ZHAO L, LIU Q, ZHANG S J, et al. Lead-free $\mathrm{AgNbO}_{3}$ antiferroelectric ceramics with an enhanced energy storage performance using $\mathrm{MnO}_{2}$ modification. J. Mater. Chem. C, 2016, 4(36): 8380-8384.

[96] MAHDI R I, MAJID W H A. Piezoelectric and pyroelectric properties of BNT-based ternary lead-free ceramic-polymer nanocomposites under different poling conditions. RSC Adv., 2016, 6(84): 81296-81309.

[97] LU Y Q, LI Y X. A review on lead-free piezoelectric ceramics studies in China. $J$. Adv. Dielect., 2011, 1(3): 269-288.

[98] PANDA P K. Review: environmental friendly lead-free piezoelectric materials. J. Mater. Sci., 2009, 44(19): 5408-5419.

[99] GAO F, DONG X L, MAO C L, et al. Energy-storage properties of $0.89 \mathrm{Bi}_{0.5} \mathrm{Na}_{0.5} \mathrm{TiO}_{3}-0.06 \mathrm{BaTiO}_{3}-0.05 \mathrm{~K}_{0.5} \mathrm{Na}_{0.5} \mathrm{NbO}_{3}$ lead-free antiferroelectric ceramics. J. Am. Ceram. Soc., 2011, 94(12): 4382 4386.

[100] DING J X, LIU Y F, LUN Y, et al. Enhanced energy-storage properties of $0.89 \mathrm{Bi}_{0.5} \mathrm{Na}_{0.5} \mathrm{TiO}_{3}-0.06 \mathrm{BaTiO}_{3}-0.05 \mathrm{~K}_{0.5} \mathrm{Na}_{0.5} \mathrm{NbO}_{3}$ lead-free anti-ferroelectric ceramics by two-step sintering method. Mater. Lett., 2014, 114: 107-110.

[101] FENG Q, YUAN C L, LIU X Y, et al. Microstructures and energy-storage properties of $(1-x)\left(\mathrm{Na}_{0.5} \mathrm{Bi}_{0.5}\right) \mathrm{TiO}_{3}-x \mathrm{BaTiO}_{3}$ with $\mathrm{BaO}-\mathrm{B}_{2} \mathrm{O}_{3}-\mathrm{SiO}_{2}$ additions. J. Mater. Sci:Mater. Electron, 2015, 26(7): 5113-5119.

[102] CHEN P, CHU B J. Improvement of dielectric and energy storage properties in $\mathrm{Bi}\left(\mathrm{Mg}_{1 / 2} \mathrm{Ti}_{1 / 2}\right) \mathrm{O}_{3}$-modified $\left(\mathrm{Na}_{1 / 2} \mathrm{Bi}_{1 / 2}\right)_{0.92} \mathrm{Ba}_{0.08} \mathrm{TiO}_{3}$ 
ceramics. J. Eur. Ceram. Soc., 2016, 36(1): 81-88.

[103] CAO W P, LI W L, DAI X F, et al. Large electrocaloric response and high energy-storage properties over a broad temperature range in lead-free NBT-ST ceramics. J. Eur. Ceram. Soc., 2016, 36(3): 593-600.

[104] XU Q, LI T M, HAO H, et al. Enhanced energy storage properties of $\mathrm{NaNbO}_{3}$ modified $\mathrm{Bi}_{0.5} \mathrm{Na}_{0.5} \mathrm{TiO}_{3}$ based ceramics. J. Eur. Ceram. Soc., 2015, 35(2): 545-553.

[105] CAO W P, LI W L, ZHANG T D, et al. High-energy storage density and efficiency of $(1-x)[0.94 \mathrm{NBT}-0.06 \mathrm{BT}]-x \mathrm{ST}$ lead-free ceramics. Energy Technol., 2015, 3(12): 1198-1204.

[106] TANG W L, XU Q, LIU H X, et al. High energy density dielectrics in lead-free $\mathrm{Bi}_{0.5} \mathrm{Na}_{0.5} \mathrm{TiO}_{3}-\mathrm{NaNbO}_{3}-\mathrm{Ba}\left(\mathrm{Zr}_{0.2} \mathrm{Ti}_{0.8}\right) \mathrm{O}_{3}$ ternary system with wide operating temperature. J. Mater. Sci: Mater Electron, 2016, 27(6): 6526-6534.

[107] LUO L H, WANG B Y, JIANG X J, et al. Energy storage properties of $(1-x)\left(\mathrm{Bi}_{0.5} \mathrm{Na}_{0.5}\right) \mathrm{TiO}_{3}-x \mathrm{KNbO}_{3}$ lead-free ceramics. J. Mater.
Sci., 2014, 49(4): 1659-1665.

[108] HAO J G, XU Z J, CHU R Q, et al. Enhanced energy-storage properties of $(1-x)\left[(1-y)\left(\mathrm{Bi}_{0.5} \mathrm{Na}_{0.5}\right) \mathrm{TiO}_{3}-y\left(\mathrm{Bi}_{0.5} \mathrm{~K}_{0.5}\right) \mathrm{TiO}_{3}\right]-x\left(\mathrm{~K}_{0 .} \mathrm{Na}_{0.5}\right)$ $\mathrm{NbO}_{3}$ lead-free ceramics. Solid State Commun, 2015, 204: 19-22.

[109] WANG Y F, LV Z L, XIE H, et al. High energy-storage properties of $\left[\left(\mathrm{Bi}_{1 / 2} \mathrm{Na}_{1 / 2}\right)_{0.94} \mathrm{Ba}_{0.06}\right] \mathrm{La}(1-x) \mathrm{Zr}_{x} \mathrm{TiO}_{3}$ lead-free anti-ferroelectric ceramics. Ceram. Int., 2014, 40(3): 4323-4326.

[110] LI F, YANG K, LIU X, et al. Temperature induced high chargedischarge performances in lead-free $\mathrm{Bi}_{1 / 2} \mathrm{Na}_{1 / 2} \mathrm{TiO}_{3}$-based ergodic relaxor ferroelectric ceramics. Scripta Mater, 2017, 141: 15-19.

[111] LI F, ZHAI J W, SHEN B, et al. Influence of structural evolution on energy storage properties in $\mathrm{Bi}_{1 / 2} \mathrm{Na}_{1 / 2} \mathrm{TiO}_{3}-\mathrm{SrTiO}_{3}-\mathrm{NaNbO}_{3}$ lead-free ferroelectric ceramics. J. Appl. Phys., 2017, 121(5): 054103-054113.

[112] MALIC B, KORUZA J, HRESCAK J, et al. Sintering of lead-free piezoelectric sodium potassium niobate ceramics. Materials, 2015, 8(12): $8117-8146$. 